\title{
Leptogenesis and Bi-Unitary Parametrization of Neutrino Yukawa Matrix
}

\author{
Kim Siyeon* \\ Institute of Physics, Academia Sinica \\ Nankang, Taipei, Taiwan 11529
}

(Dated: November 6, 2018)

\begin{abstract}
We analyze the neutrino Yukawa matrix by considering three constraints: the out-of-equilibrium condition of lepton number violating process responsible for leptogenesis, the upper bound of branching ratio of lepton flavor violating decay, and the prediction of large mixing angles using the see-saw mechanism. In a certain parametrization with bi-unitary transformation, it is shown that the structure which satisfies the constraints can be characterized by only seven types of Yukawa matrices. The constraint of the branching ratio of LFV turns out as a redundant one after applying other two constraints. We propose that this parametrization can be the framework in which the CP asymmetry of lepton number violating process can be predicted in terms of observable neutrino parameters at low energy, if necessary, under assumptions following from a theory with additional symmetries. There is an appealing model of neutrino Yukawa matrix considering the CP asymmetry for leptogenesis and the theoretical motivation to reduce the number of free parameters.
\end{abstract}

PACS numbers: $13.35 . \mathrm{Hb}, 12.15 . \mathrm{Ff}, 14.60 . \mathrm{Pq}$

*Electronic address: siyeon@phys.sinica.edu.tw 


\section{INTRODUCTION: LEPTOGENESIS}

The observed Baryon Asymmetry in Universe(BAU) can be explained with a process that satisfies three conditions: Baryon number(B) violation, Charge conjugation $(\mathrm{C})$ violation and Charge conjugation and Parity $(\mathrm{CP})$ violation, and for the B-violating process to be out-ofequilibrium [1]. Leptogenesis is a scenario for BAU in which the initial lepton asymmetry is recycled into a baryon asymmetry by the sphaleron process [2]. The initial lepton asymmetry can originate from loop-level processes involving the Yukawa couplings of Majorana neutri-

nos [3]. The baryon asymmetry $\Delta B$ can be generated after sphaleron process in thermal equilibrium washes $\Delta(B+L)$ out of $\Delta(B-L)+\Delta(B+L)$, where the $\Delta(B-L)$ is equivalent to the initial lepton asymmetry $-\Delta L[2,4,5]$. The constraints on chemical potentials of particles in thermal equilibrium establish the relations among different asymmetries. The amount of baryon asymmetry is described in terms of the ratio of particle number density to entropy density, i.e., baryon number density with respect to comoving volume element as

$$
Y_{B}=\frac{\left(n_{B}-n_{\bar{B}}\right)}{s},
$$

with entropy $s$, the range of which is $[6]$

$$
Y_{B} \approx(0.6-1) \times 10^{-10}
$$

The baryon asymmetry is related to the lepton asymmetry [5, 7] as

$$
Y_{B}=a Y_{B-L}=\frac{a}{a-1} Y_{L},
$$

where

$$
a \equiv \frac{8 N_{F}+4 N_{H}}{22 N_{F}+13 N_{H}}
$$

for example, $a=28 / 79$ for the Standard $\operatorname{Model}(\mathrm{SM})$ with three generations of fermions and a single Higgs doublet, $N_{F}=3, N_{H}=1$, and $a=8 / 23$ if $N_{F}=3, N_{H}=2$ as in supersymmetric models. Thus, the amount of the original lepton asymmetry should be not less than $\sim 10^{-9}$ to provide necessary amount of baryon asymmetry.

The generation of a lepton asymmetry also requires the CP-asymmetry and out-ofequilibrium condition. The $Y_{L}$ is explicitly parameterized by two factors, $\epsilon_{i}$, the size of $\mathrm{CP}$ asymmetry, and $\kappa$, the dilution factor from washout process.

$$
Y_{L}=\frac{\left(n_{L}-n_{\bar{L}}\right)}{s}=\kappa \frac{\epsilon_{i}}{g^{*}}
$$


where $g^{*} \simeq 110$ is the number of relativistic degree of freedom. The $\epsilon_{i}$ is the magnitude of CP asymmetry in decays of heavy Majorana neutrinos [8, 9],

$$
\epsilon_{i}=\frac{\Gamma\left(\nu_{R} \rightarrow \ell H\right)-\Gamma\left(\nu_{R} \rightarrow \ell^{c} H^{c}\right)}{\Gamma\left(\nu_{R} \rightarrow \ell H\right)+\Gamma\left(\nu_{R} \rightarrow \ell^{c} H^{c}\right)},
$$

where $i$ is 1 to 3 for generation. When one generation of the right neutrinos has a mass far below the masses for the other generations, i.e., $M_{1} \ll M_{2}, M_{3}$, the $\epsilon_{i}$ in Eq. (66) reduces to $\epsilon_{1}$ from the decay of $M_{1}$,

$$
\epsilon_{1}=\frac{3}{16 \pi} \frac{1}{\left(\mathcal{Y}_{N}^{\dagger} \mathcal{Y}_{N}\right)_{11}} \sum_{n \neq 1} \operatorname{Im}\left[\left(\mathcal{Y}_{N}^{\dagger} \mathcal{Y}_{N}\right)_{1 n}^{2}\right] \frac{M_{1}}{M_{n}}
$$

where $\mathcal{Y}_{N}$ is the matrix of Yukawa couplings in a weak basis while the right-handed Majorana mass matrix is diagonal. Such reduction to $\epsilon_{1}$ is possible since the asymmetry at $M_{3}$ was washed out by the process at $M_{2}$ and, subsequently, the asymmetry at $M_{2}$ was again washed out by the process at $M_{1}$ 10, 11, 12].

The $\kappa$ in Eq.(5) is determined by solving the full Boltzmann equations. The $\kappa$ can be simply parameterized in terms of $K$ defined as the ratio of $\Gamma_{1}$ the decay width of $\nu_{R 1}$ to $H$ the Hubble parameter at temperature $M_{1}$ [7], where $K<1$ describes processes out of thermal equilibrium and $\kappa<1$ describes washout effect,

$$
-\kappa \simeq \frac{0.3}{K(\ln K)^{0.6}}
$$

for $10 \lesssim K=\Gamma_{1} / H \lesssim 10^{6}$, and

$$
-\kappa \sim \frac{1}{2 \sqrt{K^{2}+9}}
$$

for $0 \lesssim K \lesssim 10$ [7] [13] [14]. Even if the decay is much slower than expansion rate of the universe, there is an unavoidable wash-out effect by $\kappa \sim 0.1$ [15], the size of which does not vary significantly as $K$ varies 0 to 10. Thus, the lepton asymmetry in Eq.(15) requires a lower limit in $\mathrm{CP}$ asymmetry, to say $\epsilon_{1} \gtrsim 10^{-6}$ considering $Y_{L} \gtrsim 10^{-9}$.

It has been verified that the decay processes of heavy Majorana neutrinos can generate a sufficient amount of asymmetry and the asymmetry could be kept from washout in models with neutrinos based on the see-saw mechanism [9, 14, 15, 16, 17]. The see-saw mechanism 18] links the low-energy parameters (experimental observables) to high-energy parameters (masses of SM singlet neutrinos) through 15 independent parameters of Yukawa matrix. 
Whichever the direction of prediction by see-saw mechanism goes, top-down or bottomup, the 15 parameters make full contribution in general to the prediction of any physical parameters, angles, CP phases, or mass eigenvalues at low energy or at high energy. So far, on the other hands, the lack in understanding of Yukawa parameters has restricted consideration of the low-high link so as to rely on choice of a model. For instance, there are models which predict low-energy observables in terms of parameters of leptogenesis [17, 19, 20, 21, 22] and/or minimize the degree of freedom in parameter space of leptogenesis [23] 24] at the present.

The purpose of this paper is to find the structure of matrix of neutrino Yukawa couplings (Yukawa matrix) which satisfy three constraints simultaneously: (i) the out-of-equilibrium condition of leptogenesis, (ii) the upper bound of lepton flavor violating decay in supersymmetric Standard Model (MSSM), (iii) the prediction of large mixing angle in atmospheric neutrino oscillation [25] and CHOOZ bound [26] of reactor neutrinos through see-saw mechanism. The structure of Yukawa matrix is understood as assembly of a matrix of eigenvalues, left transformation and right transformation. An advantage of the above point of view over mass structure, to say, in bi-unitary parametrization, compared with taking a whole mass structure in weak basis, is that the coexistence of any large mixing angle and hierarchical mass spectrum can be clearly defined. Another advantage is that the application of constraints can be systematic because the constraint (i) is irrelevant to the left transformation while the constraint (ii) is irrelevant to the right transformation as we will see. While the constraints (i) and (ii) have separate dependence on the right mixing and the left mixing, respectively, the constraint (iii) is connected to both left mixing and right mixing matrices. A series of phenomenological constraints were applied to the three parts of Yukawa structure carefully so as to partition parameter space into an eligible part and a ruled-out part.

First, the out-of-equilibrium condition of lepton number violating process constrains the right-handed transformation. In Section II, an appropriate parametrization for model comparison is introduced so that the size of CP asymmetry for leptogenesis can be written in a minimal way. In Section III, the out-of-equilibrium condition is rephrased in an effective form to fit out purpose and examined model by model depending on leading contribution which gives rise to comparison with the bound of the condition. Each model has its own characteristic range of elements of right transformation and the expression for the CP asymmetry from the leading contribution determined in each model. 
In Section IV, the strong bound of the branching ratio of $\mu \rightarrow e \gamma$ in MSSM is briefly reviewed. One can derive the constraint (ii) on Yukawa couplings focused on left mixing angles from renormalization group equation(RGE) of scalar mass terms. As for mixing angles, any pattern of left transformation is not ruled out by the constraint (ii). When the consideration is accompanied with the comparison between eigenvalues, a particular pattern can be ruled out by the constraint. The combination of two transformation matrices satisfied by (i) and (ii), with the type of eigenvalues, i.e., nearly degenerate or hierarchical, will be tested whether they can predict large mixing angle solution in atmospheric neutrinos and CHOOZ bound in reactor neutrinos through see-saw mechanism. We have only seven characteristic combinations which satisfy the three constraints in Tables II and III. The number of combinations is a consequence after excluding the cases where a suppressed eigenvalues can be derived only by fine tuning. There are four such cases shown in Appendix A.2. From Table $\nabla$ in Appendix A.2, we will see that the constraint (ii) is redundant if we first apply other two constraints (i) and (iii).

In Section V, we discuss the direction for the improvement of models and the possible aspect of application of further constraints in bi-unitary parametrization. A model will be examined as an appealing one for leptogenesis. A summary follows.

\section{BI-UNITARY TRANSFORMATION OF NEUTRINO YUKAWA MATRIX}

The leptogenesis has attracted attention recently because the see-saw mechanism is a plausible way for providing the scale for a lepton number violating process in the early universe. The observed neutrino mass spectrum in experiment is consistent with a see-saw model with two scales, $\Lambda_{E W}$ electroweak(EW) symmetry breaking scale and $\Lambda_{G U T}$ the scale of grand unified theory(GUT). In the low-energy effective theory, a lepton number-violating interaction results in light neutrino masses by non-zero vacuum expectation value of a Higgs scalar, and the mass matrix is diagonalized by 3 mixing angles, 1 Dirac phase and 2 Majorana phases. As far as the renormalizable gauge invariant couplings are concerned, the lepton sector will be

$$
\mathcal{L}=-\mathcal{Y}_{E} H \bar{\ell} e_{R}-\mathcal{Y}_{N} H \bar{\ell} \nu_{R}-\frac{1}{2} M_{R} \overline{\nu_{R}^{c}} \nu_{R}+\text { h.c. },
$$


in the weak basis. The physical basis of light neutrinos and heavy neutrinos where the $6 \times 6$ mass matrix is diagonal can be obtained by a $6 \times 6$ unitary transformation from the weak basis of left-handed neutrinos in the $\ell$ and right neutrinos $\nu_{R}$. This full transformation is reduced to the MNS [27] in the low-energy limit, in the basis where charged lepton mass matrix and right neutrino mass matrix $M_{R}$ are diagonal.

Considering that the Yukawa matrix is not necessarily diagonal in the physical basis where masses of Majorana neutrinos are diagonal, the transformation of Yukawa matrix (neutrino Dirac mass matrix) needs to be defined in addition to the transformation of Majorana mass. However, there might exist constraints that can connect the MNS to left-handed mixing matrix of Yukawa matrix, for example, arising from a flavor symmetry. Choose a basis where $M_{R}=\operatorname{Diag}\left(M_{1}, M_{2}, M_{3}\right)$. Let $\mathcal{Y}_{D}=\operatorname{Diag}\left(y_{1}, y_{2}, y_{3}\right)$, so that a $3 \times 3$ matrix $\mathcal{Y}_{N}$ for three-generation neutrinos can be expressed in use of bi-unitary transformation as

$$
\mathcal{Y}_{N}=\mathbb{L} \mathcal{Y}_{D} \mathbb{R}^{\dagger}
$$

Pascoli, Petcov, and Rodejohann also discussed this parametrization in Ref.[28] in comparison with other parameterizations to describe leptogenesis. The unitarity of $\mathbb{L}$ implies that any left-handed mixing angle in Yukawa matrix does not affect any parameters in leptogenesis, because the loop contribution appears in terms of $\mathcal{Y}_{N}^{\dagger} \mathcal{Y}_{N}=\mathbb{R} \mathcal{Y}_{D}^{2} \mathbb{R}^{\dagger}$.

$$
\begin{aligned}
& \left(\mathcal{Y}_{N}^{\dagger} \mathcal{Y}_{N}\right)_{11}=y_{1}^{2}\left|R_{11}\right|^{2}+y_{2}^{2}\left|R_{12}\right|^{2}+y_{3}^{2}\left|R_{13}\right|^{2}, \\
& \left(\mathcal{Y}_{N}^{\dagger} \mathcal{Y}_{N}\right)_{1 k}=y_{1}^{2} R_{11} R_{k 1}^{*}+y_{2}^{2} R_{12} R_{k 2}^{*}+y_{3}^{2} R_{13} R_{k 3}^{*},
\end{aligned}
$$

where $R_{i j}$ is an element of $\mathbb{R}$ and $k=2$ to 3. Expressing the CP asymmetry in Eq.(6) in terms of the Yukawa matrices yields,

$$
\epsilon_{1} \approx 10^{-1} \operatorname{Im}\left[\frac{\left(\mathcal{Y}_{N}^{\dagger} \mathcal{Y}_{N}\right)_{12}^{2}}{\left(\mathcal{Y}_{N}^{\dagger} \mathcal{Y}_{N}\right)_{11}} \frac{M_{1}}{M_{2}}+\frac{\left(\mathcal{Y}_{N}^{\dagger} \mathcal{Y}_{N}\right)_{13}^{2}}{\left(\mathcal{Y}_{N}^{\dagger} \mathcal{Y}_{N}\right)_{11}} \frac{M_{1}}{M_{3}}\right]
$$

and all the possible models can then be classified into three cases depending on the dominant term in Eq.(12). Factors will be indicated in bold strokes in the following if they are much smaller than order one. These lead to the following cases. However, the terms with the bold factors cannot be necessarily neglected in the leptogenesis analysis. 
Case I, where $y_{3}^{2}\left|R_{13}\right|^{2}$ is dominant,

$$
\begin{aligned}
\frac{\left(\mathcal{Y}_{N}^{\dagger} \mathcal{Y}_{N}\right)_{1 k}^{2}}{\left(\mathcal{Y}_{N}^{\dagger} \mathcal{Y}_{N}\right)_{11}} & \approx\left(\frac{y_{1}^{2} R_{11} R_{k 1}^{*}}{y_{3}\left|R_{13}\right|}+\frac{y_{2}^{2} R_{12} R_{k 2}^{*}}{y_{3}\left|R_{13}\right|}+\frac{y_{3}^{2} R_{13} R_{k 3}^{*}}{y_{3}\left|R_{13}\right|}\right)^{2} \\
& =\left(\frac{\mathbf{y}_{\mathbf{1}}\left|\mathbf{R}_{\mathbf{1 1}}\right|}{\mathbf{y}_{\mathbf{3}}\left|\mathbf{R}_{\mathbf{1 3}}\right|} y_{1} R_{k 1}^{*} e^{i \gamma_{1}}+\frac{\mathbf{y}_{\mathbf{2}}\left|\mathbf{R}_{\mathbf{1 2}}\right|}{\mathbf{y}_{\mathbf{3}}\left|\mathbf{R}_{\mathbf{1 3}}\right|} y_{2} R_{k 2}^{*} e^{i \gamma_{2}}+y_{3} R_{k 3}^{*} e^{i \gamma_{3}}\right)^{2},
\end{aligned}
$$

where $\gamma_{1}, \gamma_{2}$, and $\gamma_{3}$ are arguments of $R_{11}, R_{12}$, and $R_{13}$, respectively.

Case II, where $y_{2}^{2}\left|R_{12}\right|^{2}$ is dominant,

$$
\begin{aligned}
\frac{\left(\mathcal{Y}_{N}^{\dagger} \mathcal{Y}_{N}\right)_{1 k}^{2}}{\left(\mathcal{Y}_{N}^{\dagger} \mathcal{Y}_{N}\right)_{11}} & \approx\left(\frac{y_{1}^{2} R_{11} R_{k 1}^{*}}{y_{2}\left|R_{12}\right|}+\frac{y_{2}^{2} R_{12} R_{k 2}^{*}}{y_{2}\left|R_{12}\right|}+\frac{y_{3}^{2} R_{13} R_{k 3}^{*}}{y_{2}\left|R_{12}\right|}\right)^{2} \\
& =\left(\frac{\mathbf{y}_{\mathbf{1}}\left|\mathbf{R}_{\mathbf{1 1}}\right|}{\mathbf{y}_{\mathbf{2}}\left|\mathbf{R}_{\mathbf{1 2}}\right|} y_{1} R_{k 1}^{*} e^{i \gamma_{1}}+y_{2} R_{k 2}^{*} e^{i \gamma_{2}}+\frac{\mathbf{y}_{\mathbf{3}}\left|\mathbf{R}_{\mathbf{1 3}}\right|}{\mathbf{y}_{\mathbf{2}}\left|\mathbf{R}_{\mathbf{1 2}}\right|} y_{3} R_{k 3}^{*} e^{i \gamma_{3}}\right)^{2},
\end{aligned}
$$

Case III, where $y_{1}^{2}\left|R_{11}\right|^{2}$ is dominant,

$$
\begin{aligned}
\frac{\left(\mathcal{Y}_{N}^{\dagger} \mathcal{Y}_{N}\right)_{1 k}^{2}}{\left(\mathcal{Y}_{N}^{\dagger} \mathcal{Y}_{N}\right)_{11}} & \approx\left(\frac{y_{1}^{2} R_{11} R_{k 1}^{*}}{y_{1}\left|R_{11}\right|}+\frac{y_{2}^{2} R_{12} R_{k 2}^{*}}{y_{1}\left|R_{11}\right|}+\frac{y_{3}^{2} R_{13} R_{k 3}^{*}}{y_{1}\left|R_{11}\right|}\right)^{2} \\
& =\left(y_{1} R_{k 1}^{*} e^{i \gamma_{1}}+\frac{\mathbf{y}_{\mathbf{2}}\left|\mathbf{R}_{\mathbf{1 2}}\right|}{\mathbf{y}_{\mathbf{1}}\left|\mathbf{R}_{\mathbf{1 1}}\right|} y_{2} R_{k 2}^{*} e^{i \gamma_{2}}+\frac{\mathbf{y}_{\mathbf{3}}\left|\mathbf{R}_{\mathbf{1 3}}\right|}{\mathbf{y}_{\mathbf{1}}\left|\mathbf{R}_{\mathbf{1 1}}\right|} y_{3} R_{k 3}^{*} e^{i \gamma_{3}}\right)^{2},
\end{aligned}
$$

and Case IV, where $y_{1}^{2}\left|R_{11}\right|^{2}, y_{2}^{2}\left|R_{12}\right|^{2}$, and $y_{3}^{2}\left|R_{13}\right|^{2}$ are equally dominant,

$$
\begin{aligned}
\frac{\left(\mathcal{Y}_{N}^{\dagger} \mathcal{Y}_{N}\right)_{1 k}^{2}}{\left(\mathcal{Y}_{N}^{\dagger} \mathcal{Y}_{N}\right)_{11}} & \approx\left(\frac{y_{1}^{2} R_{11} R_{k 1}^{*}}{y_{1}\left|R_{11}\right|}+\frac{y_{2}^{2} R_{12} R_{k 2}^{*}}{y_{2}\left|R_{12}\right|}+\frac{y_{3}^{2} R_{13} R_{k 3}^{*}}{y_{3}\left|R_{13}\right|}\right)^{2} \\
& =\left(y_{1} R_{k 1}^{*} e^{i \gamma_{1}}+y_{2} R_{k 2}^{*} e^{i \gamma_{2}}+y_{3} R_{k 3}^{*} e^{i \gamma_{3}}\right)^{2}
\end{aligned}
$$

There are also cases with two equally dominant terms in $\left(\mathcal{Y}_{N}^{\dagger} \mathcal{Y}_{N}\right)_{11}$ in Eq.(12).

Case II*, where $y_{3}^{2}\left|R_{13}\right|^{2}, y_{2}^{2}\left|R_{12}\right|^{2} \gg y_{1}^{2}\left|R_{11}\right|^{2}$,

Case III*, where $y_{3}^{2}\left|R_{13}\right|^{2}, y_{1}^{2}\left|R_{11}\right|^{2} \gg y_{2}^{2}\left|R_{12}\right|^{2}$,

and Case IV*, where $y_{1}^{2}\left|R_{11}\right|^{2}, y_{2}^{2}\left|R_{12}\right|^{2} \gg y_{3}^{2}\left|R_{13}\right|^{2}$.

The corresponding expression to $\left(\mathcal{Y}_{N}^{\dagger} \mathcal{Y}_{N}\right)_{1 k}^{2} /\left(\mathcal{Y}_{N}^{\dagger} \mathcal{Y}_{N}\right)_{11}$ can be found with a single suppressed factor. Such 3 possible cases have similar aspect to the cases II, III, and IV when the out-of-equilibrium condition of lepton number violating process constrains the magnitude of $\mathbb{R}$, to be discussed further in the next section. 
The hermitian operator $\mathcal{Y}_{N}^{\dagger} \mathcal{Y}_{N}$ can be expressed with three independent CP phases and six real parameters such as

$$
\mathcal{Y}_{N}^{\dagger} \mathcal{Y}_{N}=\left(\begin{array}{ccc}
z_{11} & z_{12} e^{i \phi_{1}} & z_{13} e^{i \phi_{2}} \\
z_{12} e^{-i \phi_{1}} & z_{22} & z_{23} e^{i \phi_{3}} \\
z_{13} e^{-i \phi_{2}} & z_{23} e^{-i \phi_{3}} & z_{33}
\end{array}\right),
$$

where the $z_{i j}$ 's are real. Two phases, say $\phi_{1}$ and $\phi_{2}$, can be eliminated by a diagonal phase transformation, $\mathbb{P} \equiv \operatorname{Diag}\left(1, \exp \left(-i \phi_{1}\right), \exp \left(-i \phi_{2}\right)\right)$.

$$
\mathbb{P}^{\dagger} \mathcal{Y}_{N}^{\dagger} \mathcal{Y}_{N} \mathbb{P}=\left(\begin{array}{ccc}
z_{11} & z_{12} & z_{13} \\
z_{12} & z_{22} & z_{23} e^{i \delta^{\prime}} \\
z_{13} & z_{23} e^{-i \delta^{\prime}} & z_{33}
\end{array}\right)
$$

where $\delta^{\prime} \equiv-\phi_{1}-\phi_{2}+\phi_{3}$. In general, the diagonalization of $\mathbb{P} \mathcal{Y}_{N}^{\dagger} \mathcal{Y}_{N} \mathbb{P}^{\dagger}$ still requires 3 independent phases as combination of 2 Majorana phases and 1 Dirac phase. One can take the absolute values of the elements of $\mathbb{R}$ in Eqs.(15)-(17) to find $\left|\mathcal{Y}_{N}{ }^{\dagger} \mathcal{Y}_{N}\right|_{1 k}^{2}$, regardless of the complexity with $\gamma_{1}, \gamma_{2}$, and $\gamma_{3}$ in Eqs.(15)-(17) in terms of elements of $\mathbb{R}$. For example, the size of CP asymmetry in Eq.(14) can be written as,

$$
\begin{aligned}
\epsilon_{1} & \approx 10^{-1} \frac{\left|\mathcal{Y}_{N}^{\dagger} \mathcal{Y}_{N}\right|_{12}^{2}}{\mathcal{Y}_{N}^{\dagger} \mathcal{Y}_{N_{11}}} \sin 2 \phi_{1} \frac{M_{1}}{M_{2}} \\
& +10^{-1} \frac{\left|\mathcal{Y}_{N}^{\dagger} \mathcal{Y}_{N}\right|_{13}^{2}}{\mathcal{Y}_{N}^{\dagger} \mathcal{Y}_{N_{11}}} \sin 2 \phi_{2} \frac{M_{1}}{M_{3}}
\end{aligned}
$$

using Eq.(19).

\section{OUT-OF-EQUILIBRIUM CONDITION AND ITS CONSTRAINTS ON YUKAWA MIXING ANGLES}

The decay width of $\nu_{R 1}$ by the Yukawa interaction at tree level is

$$
\Gamma_{1}=\frac{1}{8 \pi}\left(\mathcal{Y}_{N}^{\dagger} \mathcal{Y}_{N}\right)_{11} M_{1}
$$

The out of equilibrium condition results when the Hubble parameter exceeds the decay rate, expressing Hubble parameter in terms of temperature $T$,

$$
\Gamma_{1}<H=1.66 g_{*}^{1 / 2} \frac{T^{2}}{M_{p l}} .
$$


At temperature $T=M_{1}$, the condition can be rephrased as,

$$
\left(\mathcal{Y}_{N}^{\dagger} \mathcal{Y}_{N}\right)_{11} \lesssim \zeta_{1}^{2}
$$

where

$$
\zeta_{1}^{2} \equiv 10^{2} \frac{M_{1}}{M_{p l}}
$$

The upper bound $\zeta_{1}^{2}$ in Eq.(24) cannot exceed $M_{1} / M_{G U T}$, when $M_{G U T}$ is chosen in a range $\left(10^{-4}-10^{-3}\right) M_{P l}$ depending on a theoretical framework and the masses of heavy Majorana neutrinos cannot be higher than $M_{G U T}$. As long as $M_{1}$ is hierarchically smaller than the other masses of heavy neutrinos, as assumed here, the $\zeta_{1}^{2}$ should be considered safely as small as order of $\lesssim 10^{-2}$.

The condition was examined in detail by Buchmuller and Plumacher [12] with the relation of generated $B-L$ asymmetry to the effective mass defined as

$$
\tilde{m}_{1}=\left(\mathcal{Y}_{N}^{\dagger} \mathcal{Y}_{N}\right)_{11} \frac{v_{2}^{2}}{M_{1}}
$$

Besides the upper bound which keeps the asymmetry protected from wash-out effect, there is also a lower bound for $\tilde{m}_{1}$ since, at high temperature, weak Yukawa couplings cannot produce enough neutrinos. The eligible $\tilde{m}_{1}$ capable of generating sufficient $B-L$ (or equivalently $L)$ asymmetry, lies in the range $m_{1}<\tilde{m}_{1}<m_{3}$ due to see-saw mechanism [17] [21], where $m_{1}$ is the mass of the lightest neutrino, which is presumed to be non-zero but smaller than $m_{2} \approx \sqrt{\triangle m_{\odot}^{2}}$ and $m_{3}$ is the mass of the heaviest light neutrino whose magnitude is about $\sqrt{\triangle m_{\text {atm }}^{2}}$, assuming a hierarchical light neutrino spectrum.

Using Eq.(12), the condition in Eq.(24) can be rephrased in such a convenient way as

$$
y_{1}^{2}\left|R_{11}\right|^{2}+y_{2}^{2}\left|R_{12}\right|^{2}+y_{3}^{2}\left|R_{13}\right|^{2} \lesssim \zeta_{1}^{2}
$$

The first significant implication of the above condition is

$$
\left|R_{13}\right| \lesssim \zeta_{1}
$$

when $y_{3}$ is of order one. The hierarchy in masses of right-handed neutrinos is a preliminary for the CP asymmetry in Eq.(17) of leptogenesis, causing the smallness of $\zeta_{1}$ and so a smallangle constraint for $\left|R_{13}\right|$. The next implication is the smallness of $y_{1}$,

$$
y_{1} \lesssim \zeta_{1}
$$


TABLE I: Model classification: Cases I, II, III, and IV are introduced in Eqs. (15, 18). Case -a is for $y_{3} \gg y_{2} \gg y_{1}$, Case - $\mathbf{b}$ for $y_{3} \sim y_{2} \gg y_{1}$, and Case -c for $y_{3} \gg y_{2} \sim y_{1}$. In $|\mathbb{R}|, \zeta_{1}$ is defined in Eq.(24), $\zeta_{s}$ 's in Cases I-b, IV-b and in Case II-b represent small values of $\left|R_{13}\right|$ and $\left|R_{12}\right|$, respectively, while $\zeta_{s}$ in Case III and Case IV-a represent simply a small angle. The $s_{12}$ and $s_{23}$ imply that those angles can be large or small. The Case II and the Case -c cannot be compatible with each other.

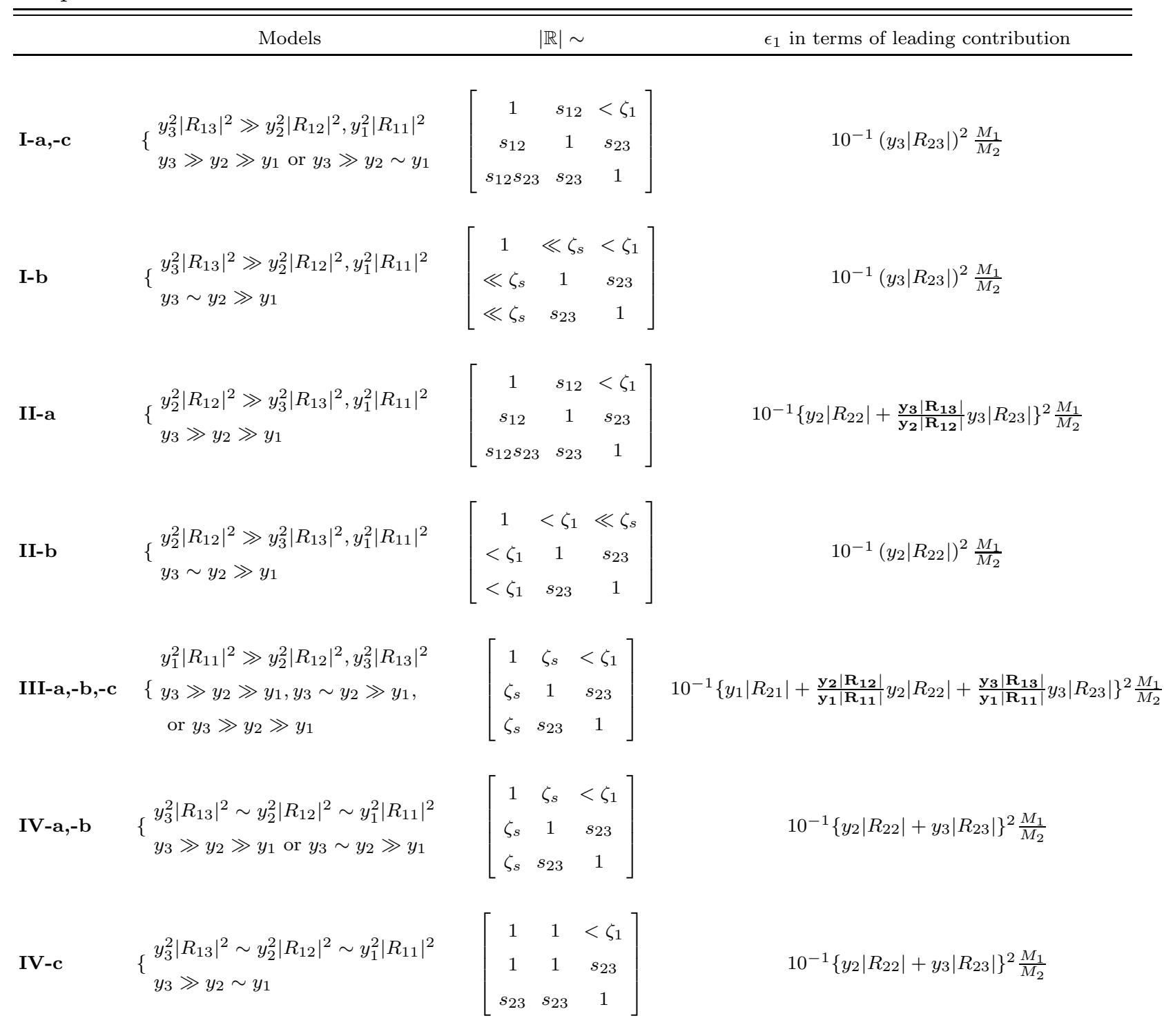

since $\left|R_{11}\right|$ is always of order one whether mixing angles are large or small. So $y_{1}$ cannot be of the same approximate size as $y_{3}$ if $y_{3} \sim 1$. The models that satisfy the two conditions in Eqs.(28)-(29) can be according to which term dominates in the real number $\left(\mathcal{Y}_{N}^{\dagger} \mathcal{Y}_{N}\right)_{11}$ 
and how different the eigenvalues $y_{1}, y_{2}$, and $y_{3}$ of the Yukawa matrix are. A model with $y_{3} \gg y_{2} \gg y_{1}$ is denoted as Case -a, the one with $y_{3} \sim y_{2} \gg y_{1}$ as Case - $\mathbf{b}$, and the one with $y_{3} \gg y_{2} \sim y_{1}$ as Case -c. The leading contribution to $\epsilon_{1}$ in Table $\llbracket$ is presented by the first term in Eq.(14).

In Case I in Eq.(15), if the eigenvalues are of normal hierarchy, $y_{3} \gg y_{2}$, the out-ofequilibrium condition in Eq.(27) does not place any constraints on elements in $\mathbb{R}$ other than $\left|R_{13}\right|<\zeta_{1}$, while nearly degenerate eigenvalues, $y_{3} \sim y_{2}$, restrict $\left|R_{12}\right|$ to be small. If the transformation $\mathbb{R} / \mathbb{J} \equiv \mathbb{R} \mathbb{J}^{-1}$ is parameterized as

$$
\mathbb{R} / \mathbb{J}=\left(\begin{array}{ccc}
c_{13} c_{12} & s_{12} c_{13} & s_{13} \\
-s_{12} c_{23}-s_{23} s_{13} c_{12} e^{-i \delta} & c_{23} c_{12}-s_{23} s_{13} s_{12} e^{-i \delta} & s_{23} c_{13} e^{-i \delta} \\
s_{23} s_{12} e^{i \delta}-s_{13} c_{23} c_{12} & -s_{23} c_{12} e^{i \delta}-s_{13} s_{12} c_{23} & c_{23} c_{13}
\end{array}\right)
$$

where $\mathbb{J}$ is a diagonal phase transformation, one can deduce the smallness of $\left|R_{21}\right|$ and $\left|R_{31}\right|$ from the smallness of $R_{12}$ due to small $s_{12}$. The possible structures of $\mathbb{R}$ for the Case $\mathbf{I}$ are listed in Table I where $s_{12}$ and $s_{23}$ specified in the table are allowed to be either small angle or large one, while $\lambda_{s}$ indicates that only a small angle is allowed. If $s_{23}$ is not so small, the leading contribution of the imaginary part which leads to $\epsilon_{1}$ consists of mainly $\left(y_{3}\left|R_{23}\right|\right)^{2}$ and $M_{1} / M_{2}$. The model examined in Ref.[17] is an example of Case I-a.

In Case II, in Eq.(16), if the eigenvalues are of normal hierarchy with $y_{3} \gg y_{2}$, the out-of-equilibrium condition allows $\left|R_{12}\right|$ to be much larger than $\left|R_{13}\right|$. If the assumption is made that $y_{2}$ is not larger than $\zeta_{1}$, then $\left|R_{12}\right|$ can still be a large angle. In other words, $\left|R_{12}\right|$ can be either small or large, though, it is larger than $\zeta_{1}$ in this model. The leading contribution to $\epsilon_{1}$ depends on mainly from

$$
y_{2}\left|R_{22}\right|+\frac{\mathbf{y}_{\mathbf{3}}\left|\mathbf{R}_{\mathbf{1 3}}\right|}{\mathbf{y}_{\mathbf{2}}\left|\mathbf{R}_{\mathbf{1 2}}\right|} y_{3}\left|R_{23}\right| .
$$

Otherwise, the two eigenvalues, $y_{2}$ and $y_{3}$ in Case II, are close to each other. The element $\left|R_{12}\right|$ is smaller than $\zeta_{1}$ and $\left|R_{13}\right|$ is far smaller than $\left|R_{12}\right|$. The term $\left|\mathcal{Y}_{N}^{\dagger} \mathcal{Y}_{N}\right|_{12}^{2} /\left(\mathcal{Y}_{N}^{\dagger} \mathcal{Y}_{N}\right)_{11}$ reduces to $y_{2}\left|R_{22}\right|$ which is now the leading contribution to $\epsilon_{1}$ of order one. The assumption implies $\left|R_{12}\right| \gg y_{1} / y_{2}$, so that the Case II-c with $y_{1} \sim y_{2}$ is ruled out. For the same reason, there can not be Case $\mathbf{I I}^{*}$-c, while Case $\mathbf{I I}^{*}$-a with $y_{3} \gg y_{2}$ and Case II*-b with $y_{3} \sim y_{2}$ and small $\left|R_{12}\right|$ are allowed. The allowed ranges in the elements of $|\mathbb{R}|$ for the Case 
II*-a and Case II*-b can be obtained as the same as in the Case II-a and Case II-b, respectively.

Case III in Eq.(17) corresponds to $\left|R_{12}\right| \ll y_{1} / y_{2}$, i.e., $\left|R_{12}\right|$ is always a small angle. Any part of the contribution to $\left|\mathcal{Y}_{N}^{\dagger} \mathcal{Y}_{N}\right|_{12}^{2} /\left(\mathcal{Y}_{N}^{\dagger} \mathcal{Y}_{N}\right)_{11}$ is not yet ruled out by the assumptions given here. The Case III* also gives rise to similar constraint on $\left|R_{12}\right|$ as Case III does.

In Case IV in Eq.(18), a subcase with $y_{3} \sim y_{2}$ and $\left|R_{12}\right|$ is not allowed by out-ofequilibrium condition because $y_{2}\left|R_{12}\right|$ should not be larger than $\zeta_{1}$. This is the case with $\left|R_{12}\right| \sim y_{1} / y_{2}$. Thus, $\left|R_{12}\right|$ is small for $y_{2} \gg y_{1}$, while $\left|R_{12}\right|$ is large for $y_{2} \sim y_{1}$. The possible structures of $|\mathbb{R}|$ derived in Case $\mathbf{I V}^{*}$ also is consistent withe those in Case IV.

The structures of $|\mathbb{R}|$ in Cases II*, III*, IV* attribute their correspondences to those in Cases II, III, IV, respectively, to relative significance in comparison of $y_{1}\left|R_{11}\right|$ and $y_{2}\left|R_{12}\right|$ when $y_{3}\left|R_{13}\right|$ is fixed. In other word, once $\left|R_{13}\right|$ is bounded, cases can be effectively classified into a case with $y_{1}\left|R_{11}\right| \ll y_{2}\left|R_{12}\right|$, a case with $y_{2}\left|R_{12}\right| \ll y_{1}\left|R_{11}\right|$, and a case with $y_{1}\left|R_{11}\right| \sim y_{2}\left|R_{12}\right|$. Thus, the cases with two equal dominant terms can remain without further detailed discussion.

In Table 【 where the various cases are considered, the allowed structures of $|\mathbb{R}|$ under those cases and the $\mathrm{CP}$ asymmetry $\epsilon_{1}$ in terms of leading contribution after ruling out the suppressed part under the assumptions are summarized. In Case I and Case IV with $\left|R_{23}\right|$ of order one , or in Case II-b, $\epsilon_{1}$ can reach its model independent maximum value,

$$
\epsilon_{1 \max } \sim 10^{-1} \frac{M_{1}}{M_{2}}
$$

where $M_{1}<M_{2}$.

\section{LOW-ENERGY OBSERVABLES AND THEIR CONSTRAINTS ON YUKAWA MIXING TRANSFORMATIONS}

The current experimental limit on the branching $\operatorname{ratio}(B R)$ of the lepton flavor violating (LFV) decay mode, $\mu \rightarrow e \gamma$ is

$$
B R(\mu \rightarrow e \gamma)<1.2 \times 10^{-11}
$$

In the SM, the branching ratio is $<10^{-50}$ suppressed far below the observable bound [29]. Another theoretical framework where one can estimate the size of the LFV decay rates 
is the minimal Supersymmetry(SUSY) Standard Model(MSSM), where the rates for LFV processes can be enhanced due to large $\tan \beta$ [30]. The neutrino Yukawa couplings cause the renormalization group equations (RGE) to develop flavor changing contribution to soft masses of scalar leptons, which may originate at a universal value for all kinds scalar leptons at GUT scale $M_{X}$. The generated LFV mass terms for scalar leptons after integrating RGE is

$$
\begin{aligned}
\left(\triangle m_{\tilde{L}}^{2}\right)_{i j} & \approx \frac{-1}{8 \pi^{2}}\left(3 m_{0}^{2}+A_{0}^{2}\right) \sum_{k} \mathcal{Y}_{N i k} \log \frac{M_{X}}{M_{k}} \mathcal{Y}_{N k j}^{\dagger} \\
& \sim \frac{-1}{8 \pi^{2}}\left(3 m_{0}^{2}+A_{0}^{2}\right) \log \frac{M_{X}}{M_{3}}\left(\mathcal{Y}_{N} \mathcal{Y}_{N}^{\dagger}\right)_{i j},
\end{aligned}
$$

where $\log \frac{M_{X}}{M_{k}}$ for $k=1-3$ can be considered as of the same order even when $M_{k}$ 's are of hierarchy. The $m_{0}^{2}$ and $A_{0}^{2}$ are the universal masses and universal trilinear couplings, respectively, in soft SUSY-breaking lagrangian [30]. The flavor changing decay in Eq.(33) involves neutralino exchange and chargino exchange in loop diagrams, and the resulting amplitude is proportional to $\tan \beta$. Therefore the branching ratios can be expressed in terms of $\mathcal{Y}_{N} \mathcal{Y}_{N}^{\dagger}$ as follows;

$$
\begin{aligned}
& B R\left(\ell_{i} \rightarrow \ell_{j} \gamma\right) \sim \\
& \frac{\alpha^{3}}{G_{F}^{2} m_{s}^{8}}\left|\frac{-1}{8 \pi^{2}}\left(3 m_{0}^{2}+A_{0}^{2}\right) \log \frac{M_{X}}{M_{3}}\left(\mathcal{Y}_{N} \mathcal{Y}_{N}^{\dagger}\right)_{i j}\right|^{2} \tan ^{2} \beta,
\end{aligned}
$$

where $m_{s}$ is the typical mass of a superparticle, $\alpha$ is the fine structure constant, and $G_{F}$ is the Fermi constant 30] 31]. Recently, LFV in low-energy has been considered for its relevance with leptogenesis [24] [32].

Even if a model can predict an enhanced branching ratio near the range accessible in a near future experiment, the eligible structure of Yukawa matrix implies there might be a constraint for severely suppressed mixing angles in left-handed transformation of Yukawa matrix at low energy. The enhancement mechanism of branching ratios is possible not only by raising $\tan \beta$ but also by increasing mixing angles. Hisano et al [30]. analyzed the CKM matrix of quark mixing. With small mixing angles as in CKM, the branching ratios of $\ell_{i} \rightarrow \ell_{j} \gamma$ processes for large $\tan \beta$ can reach close to the current experimental bounds in Eq.(33). Considering the strong upper bound in current experiment limit for the decay mode $\mu \rightarrow e \gamma$, the relevant constraint on the Yukawa couplings is, in terms of the elements 
of $\mathbb{L}$ defined in Eq.(11),

$$
\begin{aligned}
& \left(\mathcal{Y}_{N} \mathcal{Y}_{N}^{\dagger}\right)_{21}= \\
& y_{1}^{2} L_{21} L_{11}^{*}+y_{2}^{2} L_{22} L_{12}^{*}+y_{3}^{2} L_{23} L_{13}^{*} \ll 1
\end{aligned}
$$

which can be satisfied only if $y_{2}$ or $\left|L_{12}\right|$ is small and $\left|L_{23}\right|$ or $\left|L_{13}\right|$ is small.

In the rest of this section, I combine the constraint on $\mathbb{R}$ from the out-of-equilibrium condition and the constraint on $\mathbb{L}$ from the upper bound of the branching ratio of $\mu \rightarrow e \gamma$. The question is whether the result of those combinations can be accommodated with the experimentally observed large mixing angles of light neutrinos when implemented via seesaw mechanism. Light neutrino masses can be obtained through the see-saw mechanism where Dirac masses are defined in terms of the non-zero vacuum expectation value $v_{2}$ of a light Higgs in MSSM with two Higgs doublets,

$$
\begin{aligned}
m_{\nu} & =-v_{2}^{2} \mathcal{Y}_{N} M_{R}^{-1} \mathcal{Y}_{N}^{T}, \\
& =-v_{2}^{2} \mathbb{L} \mathcal{Y}_{D} \mathbb{R}^{\dagger} M_{R}^{-1} \mathbb{R}^{*} \mathcal{Y}_{D}^{T} \mathbb{L}^{T}
\end{aligned}
$$

In this basis the mass matrix of charged leptons is diagonal. When the transformations $\mathbb{L}$ and $\mathbb{R}$ are trivial, i.e., equal to the identity, the see-saw mechanism yields the light neutrino mass matrix,

$$
m_{\nu}=-v_{2}^{2} \operatorname{Diag}\left(\frac{y_{1}^{2}}{M_{1}}, \frac{y_{2}^{2}}{M_{2}}, \frac{y_{3}^{2}}{M_{3}}\right)
$$

from Eq.(37). It will be interesting to watch how neutrino mass matrix in Eq.(37) can change as the type of transformation matrix $\mathbb{R}$, and subsequently $\mathbb{L}$, switches from one to another

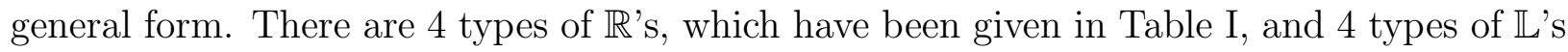
are as follows:

$$
\begin{aligned}
& \left|\mathbb{R}_{1}\right| \sim\left(\begin{array}{lll}
1 & \rho & \rho \\
\rho & 1 & 1 \\
\rho & 1 & 1
\end{array}\right),\left|\mathbb{L}_{1}\right| \sim\left(\begin{array}{lll}
1 & \lambda & \lambda \\
\lambda & 1 & 1 \\
\lambda & 1 & 1
\end{array}\right), \\
& \left|\mathbb{R}_{2}\right| \sim\left(\begin{array}{lll}
1 & 1 & \rho \\
1 & 1 & \rho \\
\rho & \rho & 1
\end{array}\right),\left|\mathbb{L}_{2}\right| \sim\left(\begin{array}{lll}
1 & 1 & \lambda \\
1 & 1 & \lambda \\
\lambda & \lambda & 1
\end{array}\right),
\end{aligned}
$$




$$
\begin{gathered}
\left|\mathbb{R}_{3}\right| \sim\left(\begin{array}{lll}
1 & 1 & \rho \\
1 & 1 & 1 \\
1 & 1 & 1
\end{array}\right),\left|\mathbb{L}_{3}\right| \sim\left(\begin{array}{lll}
1 & 1 & \lambda \\
1 & 1 & 1 \\
1 & 1 & 1
\end{array}\right), \\
\left|\mathbb{R}_{4}\right| \sim\left(\begin{array}{lll}
1 & \rho & \rho \\
\rho & 1 & \rho \\
\rho & \rho & 1
\end{array}\right),\left|\mathbb{L}_{4}\right| \sim\left(\begin{array}{lll}
1 & \lambda & \lambda \\
\lambda & 1 & \lambda \\
\lambda & \lambda & 1
\end{array}\right),
\end{gathered}
$$

which satisfy the out-of-equilibrium condition in Eq.(24). The most simplified notation is used, which is that diagonal elements and large mixing elements are denoted by 1 , whereas small mixing elements are denoted by arbitrary small values by $\rho \ll 1$ and $\lambda \ll 1$ in $\mathbb{R}$ and $\mathbb{L}$, respectively. That is, there are only two kinds of elements in a transformation matrix: order one or far less than order one. The more detailed $\mathbb{R}$ 's have been sorted in Table $\mathbb{\square}$ by considering out-of-equilibrium condition. Later, in case the leading order in an entry of light neutrino mass matrix originates from small angles in transformation matrices, the light neutrino mass matrices in terms of the small angles, considering the actual entries as expansion of small angles, are shown for models in Appendix A.1.

Each structure of $\left|\mathbb{L}_{1}\right|-\left|\mathbb{L}_{4}\right|$ is similar to each of $\left|\mathbb{R}_{1}\right|-\left|\mathbb{R}_{4}\right|$, as shown in Eq.(39), which satisfy the condition in Eq. (36) which is the same form as Eq.(27) for the $\mathbb{R}$ matrix. For the suppressed $\left(\mathcal{Y}_{N} \mathcal{Y}_{N}^{\dagger}\right)_{21}$, it is necessary that $L_{12}$ and $L_{13} L_{23}$ be small for $y_{2} \sim y_{3}$, while the smallness of $L_{12}$ or $L_{23}$ is optional for $y_{2} \ll y_{3}$ and $L_{13}$ small. The possibility of large $\left|L_{13}\right|$ is excluded by the CHOOZ bound [26] on the MNS neutrino mixing matrix.

In Tables II and III, all the possible structures of light neutrino mass matrix, which can be produced by Eq.(37) using $\mathbb{R}^{\prime}$ 's and $\mathbb{L}$ 's in Eq.(39), are listed. Due to $M_{1} \ll M_{2}, M_{3}$ assumed for Eq.(17), the following approximation can be used in the tables.

$$
\begin{aligned}
& M_{123}^{-1} \sim M_{12}^{-1} \sim M_{1}^{-1}, \\
& M_{123}^{-1} \gg M_{23}^{-1}, M_{123}^{-1} \gg M_{3}^{-1},
\end{aligned}
$$

where

$$
\begin{aligned}
& M_{123}^{-1} \equiv M_{1}^{-1}+M_{2}^{-1}+M_{3}^{-1}, \\
& M_{12}^{-1} \equiv M_{1}^{-1}+M_{2}^{-1},
\end{aligned}
$$


TABLE II: List of possible structures of matrix $m_{\nu} / v_{2}^{2}$ which can be produced by see-saw mechanism in Eq.(37) using $\mathbb{R}^{\prime}$ 's and $\mathbb{L}$ 's in Eq.(39), where $m_{\nu}$ is a matrix mass of light neutrinos and $v_{2}$ is a vacuum expectation value in two-Higgs-doublet models. $M_{123}$ and $M_{12}$ are defined in Eq.(41) and $y_{123}$ and $y_{12}$ are defined in Eq.(42). Please see Appendix A.1 for the entries marked by *.

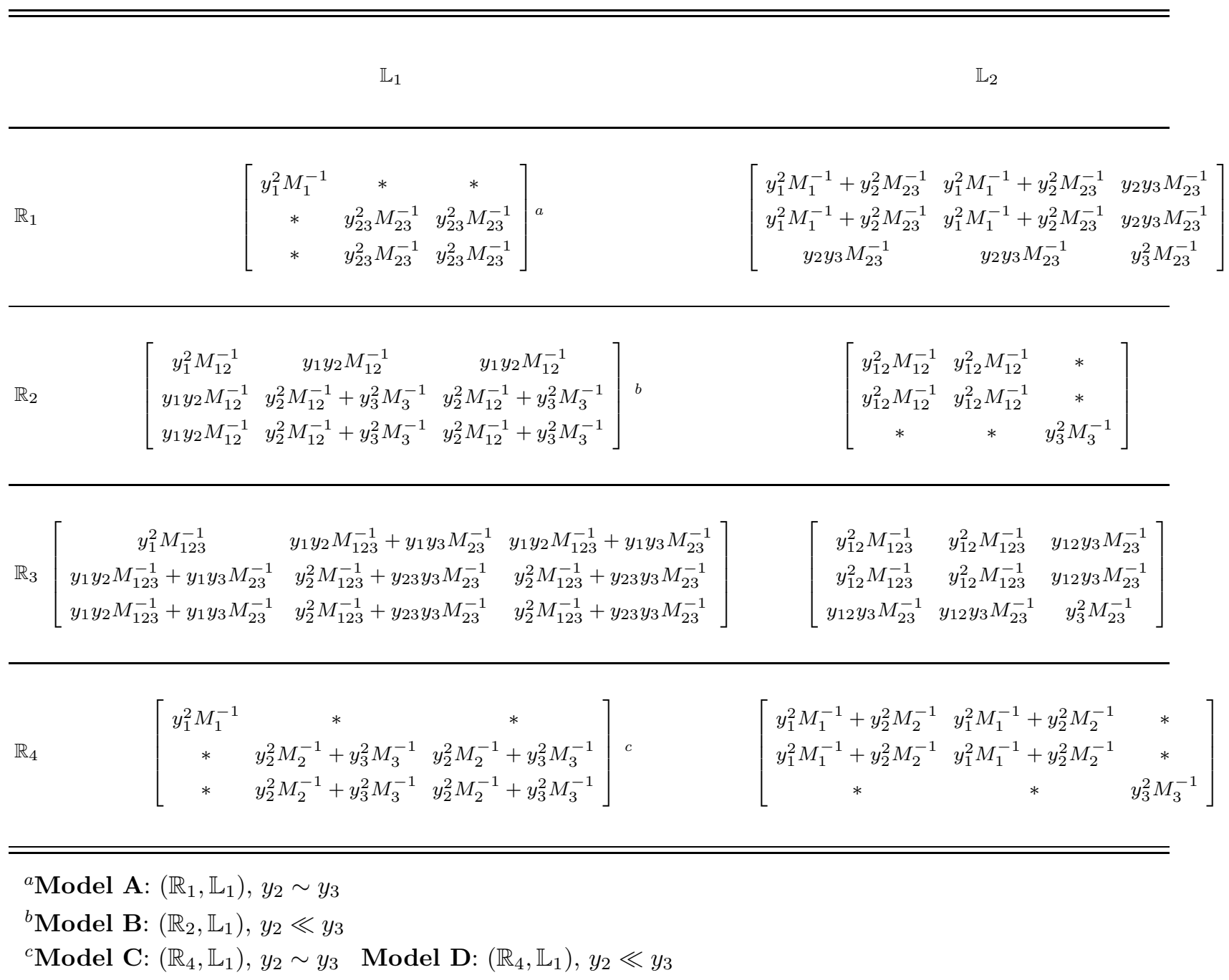

etc., and

$$
\begin{aligned}
& y_{123}=y_{1}+y_{2}+y_{3}, \\
& y_{12}=y_{1}+y_{2}, \quad y_{23}=y_{2}+y_{3} .
\end{aligned}
$$

The comparison between $M_{2}$ and $M_{3}$ is not yet fixed. Either possibility, $M_{2} \ll M_{3}$ or $M_{2} \sim M_{3}$, is still considered.

The structures listed in Tables \I and III are investigated whether they can predict two large mixing angles and one small mixing angle in light neutrinos. As for the large mixing 
TABLE III: The matrix $m_{\nu} / v_{2}^{2}$ which can be produced by see-saw mechanism in Eq.(37) using $\mathbb{R}$ 's and $\mathbb{L}$ 's in Eq.(39). The $i$ in the matrix from $\mathbb{R}_{4}$ and $\mathbb{L}_{3}$ runs 1 to 3. Please see Appendix A.1 for the entries marked by $*$.

\begin{tabular}{|c|c|c|}
\hline & $\mathbb{L}_{3}$ & $\mathbb{L}_{4}$ \\
\hline $\mathbb{R}_{1}$ & {$\left[\begin{array}{ccc}y_{1}^{2} M_{1}^{-1}+y_{2}^{2} M_{23}^{-1} & y_{1}^{2} M_{1}^{-1}+y_{2} y_{23} M_{23}^{-1} & y_{1}^{2} M_{1}^{-1}+y_{2} y_{23} M_{23}^{-1} \\
y_{1}^{2} M_{1}^{-1}+y_{2} y_{23} M_{23}^{-1} & y_{1}^{2} M_{1}^{-1}+y_{23}^{2} M_{23}^{-1} & y_{1}^{2} M_{1}^{-1}+y_{23}^{2} M_{23}^{-1} \\
y_{1}^{2} M_{1}^{-1}+y_{2} y_{23} M_{23}^{-1} & y_{1}^{2} M_{1}^{-1}+y_{23}^{2} M_{23}^{-1} & y_{1}^{2} M_{1}^{-1}+y_{23}^{2} M_{23}^{-1}\end{array}\right]$} & $\left.\begin{array}{ccc}y_{1}^{2} M_{1}^{-1} & * & * \\
* & y_{2}^{2} M_{23}^{-1} & y_{2} y_{3} M_{23}^{-1} \\
* & y_{2} y_{3} M_{23}^{-1} & y_{3}^{2} M_{23}^{-1}\end{array}\right]$ \\
\hline $\mathbb{R}_{2}$ & {$\left[\begin{array}{ccc}y_{12}^{2} M_{12}^{-1} & y_{12}^{2} M_{12}^{-1} & y_{12}^{2} M_{12}^{-1} \\
y_{12}^{2} M_{12}^{-1} & y_{12}^{2} M_{12}^{-1}+y_{3}^{2} M_{3}^{-1} & y_{12}^{2} M_{12}^{-1}+y_{3}^{2} M_{3}^{-1} \\
y_{12}^{2} M_{12}^{-1} & y_{12}^{2} M_{12}^{-1}+y_{3}^{2} M_{3}^{-1} & y_{12}^{2} M_{12}^{-1}+y_{3}^{2} M_{3}^{-1}\end{array}\right] b$} & {$\left[\begin{array}{ccc}y_{1}^{2} M_{12}^{-1} & y_{1} y_{2} M_{12}^{-1} & * \\
y_{1} y_{2} M_{12}^{-1} & y_{2}^{2} M_{12}^{-1} & * \\
* & * & y_{3}^{2} M_{3}^{-1}\end{array}\right]$} \\
\hline $\mathbb{R}_{3}$ & $\begin{array}{ccc}y_{12}^{2} M_{123}^{-1} & y_{12}^{2} M_{123}^{-1}+y_{12} y_{3} M_{23}^{-1} & y_{12}^{2} M_{123}^{-1}+y_{12} y_{3} M_{23}^{-1} \\
y_{12}^{2} M_{123}^{-1}+y_{12} y_{3} M_{23}^{-1} & y_{12}^{2} M_{1}^{-1}+y_{123}^{2} M_{23}^{-1} & y_{12}^{2} M_{1}^{-1}+y_{123}^{2} M_{23}^{-1} \\
y_{12}^{2} M_{123}^{-1}+y_{12} y_{3} M_{23}^{-1} & y_{12}^{2} M_{1}^{-1}+y_{123}^{2} M_{23}^{-1} & y_{12}^{2} M_{1}^{-1}+y_{123}^{2} M_{23}^{-1}\end{array}$ & $\begin{array}{ccc}y_{1}^{2} M_{123}^{-1} & y_{1} y_{2} M_{123}^{-1} & y_{1} y_{3} M_{23}^{-1} \\
y_{1} y_{2} M_{123}^{-1} & y_{2}^{2} M_{123}^{-1} & y_{2} y_{3} M_{23}^{-1} \\
y_{1} y_{3} M_{23}^{-1} & y_{2} y_{3} M_{23}^{-1} & y_{3}^{2} M_{23}^{-1}\end{array}$ \\
\hline $\mathbb{R}_{4}$ & $\left.\begin{array}{ccc}y_{1}^{2} M_{1}^{-1}+y_{2}^{2} M_{2}^{-1} & y_{1}^{2} M_{1}^{-1}+y_{2}^{2} M_{2}^{-1} & y_{1}^{2} M_{1}^{-1}+y_{2}^{2} M_{2}^{-1} \\
y_{1}^{2} M_{1}^{-1}+y_{2}^{2} M_{2}^{-1} & y_{i}^{2} M_{i}^{-1} & y_{i}^{2} M_{i}^{-1} \\
y_{1}^{2} M_{1}^{-1}+y_{2}^{2} M_{2}^{-1} & y_{i}^{2} M_{i}^{-1} & y_{i}^{2} M_{i}^{-1}\end{array}\right] c$ & {$\left[\begin{array}{ccc}y_{1}^{2} M_{1}^{-1} & * & * \\
* & y_{2}^{2} M_{2}^{-1} & * \\
* & * & y_{3}^{2} M_{3}^{-1}\end{array}\right]$} \\
\hline
\end{tabular}

${ }^{a}$ Model E: $\left(\mathbb{R}_{1}, \mathbb{L}_{4}\right), y_{2} \sim y_{3}$

${ }^{b}$ Model F: $\left(\mathbb{R}_{2}, \mathbb{L}_{3}\right), y_{2} \ll y_{3}$

${ }^{c}$ Model G: $\left(\mathbb{R}_{4}, \mathbb{L}_{3}\right), y_{2} \ll y_{3}$

angle in atmospheric neutrino oscillation, the ratio of two elements $m_{\nu 23} / m_{\nu 33}$ must not be less than order one. On the other hand, for the ratio $m_{\nu 12} / m_{\nu 22}$ to be not less than order one is a sufficient condition for the large mixing angle in solar neutrino oscillation, because there is still a room for a kind of manipulation including fine tuning so as to make the mixing angle large even without the naive ratio $m_{\nu 12} / m_{\nu 22}$. The application of the constraints from light neutrino mass spectrum is described more in Appendix A.2. When $y_{2} \sim y_{3}$, the combination of $\mathbb{R}$ and $\mathbb{L}$ which help us predict large mixing angle for atmospheric neutrinos is $\left(\mathbb{R}_{1}, \mathbb{L}_{1}\right)$ for Model $\mathbf{A},\left(\mathbb{R}_{4}, \mathbb{L}_{1}\right)$ for Model $\mathbf{C}$, or $\left(\mathbb{R}_{1}, \mathbb{L}_{4}\right)$ for Model $\mathbf{E}$ where $\mathbb{R}_{i}$ and $\mathbb{L}_{i}$ with $i=1-4$ are defined in Eqs.(39). The solutions $\mathbb{R}_{2}$ or $\mathbb{R}_{3}$ are not included since the out-of-equilibrium 
TABLE IV: List of phenomenologically viable models of Yukawa matrices and the light neutrino mass matrix divided by $v^{2}$ derived through see-saw mechanism. The eigenvalues in Models A, $\mathbf{C}$, and $\mathbf{E}$ are of $y_{2} \ll y_{3} \sim 1$, while those in Models $\mathbf{B}, \mathbf{D}, \mathbf{F}$, and $\mathbf{G}$ are of $y_{1} \ll y_{2} \sim y_{3} \sim 1$. The $y_{2}$ and $y_{3}$ specified in $m_{\nu} / v^{2}$ remain for a later use.

\begin{tabular}{|c|c|c|c|c|}
\hline Model & $|\mathbb{L}|$ & $\mathcal{Y}_{D}$ & $\left|\mathbb{R}^{\dagger}\right|$ & $m_{\nu} / v^{2} \sim \mathbb{L} \mathcal{Y}_{D} \mathbb{R}^{\dagger} M_{R}^{-1} \mathbb{R}^{*} \mathcal{Y}_{D}^{T} \mathbb{L}^{T}$ \\
\hline A & $\left.\begin{array}{lll}1 & \lambda & \lambda \\
\lambda & 1 & 1 \\
\lambda & 1 & 1\end{array}\right]$ & $\left.\begin{array}{lll}y_{1} & & \\
& 1 & \\
& & \\
& & 1\end{array}\right]$ & $\begin{array}{lll}1 & \rho & \rho \\
\rho & 1 & 1 \\
\rho & 1 & 1\end{array}$ & $\left.\begin{array}{ccc}y_{1}^{2} M_{1}^{-1} & \sqrt{ } & \sqrt{ } \\
\left(y_{1}^{2} M_{1}^{-1}+y_{23}^{2} M_{23}^{-1}\right) \lambda+y_{1} y_{23} M_{123}^{-1} \rho & y_{23}^{2} M_{23}^{-1} & \sqrt{ } \\
\left(y_{1}^{2} M_{1}^{-1}+y_{23}^{2} M_{23}^{-1}\right) \lambda+y_{1} y_{23} M_{123}^{-1} \rho & y_{23}^{2} M_{23}^{-1} & y_{23}^{2} M_{23}^{-1}\end{array}\right]$ \\
\hline B & $\left.\begin{array}{lll}1 & \lambda & \lambda \\
\lambda & 1 & 1 \\
\lambda & 1 & 1\end{array}\right]$ & $\begin{array}{lll}y_{1} & & \\
& & \\
& y_{2} & \\
& & 1\end{array}$ & {$\left[\begin{array}{lll}1 & 1 & \rho \\
1 & 1 & \rho \\
\rho & \rho & 1\end{array}\right]$} & $\left.\begin{array}{ccc}y_{1}^{2} M_{12}^{-1} & \sqrt{ } & \sqrt{ } \\
y_{1} y_{2} M_{12}^{-1} & y_{2}^{2} M_{12}^{-1}+y_{3}^{2} M_{3}^{-1} & \sqrt{ } \\
y_{1} y_{2} M_{12}^{-1} & y_{2}^{2} M_{12}^{-1}+y_{3}^{2} M_{3}^{-1} & y_{2}^{2} M_{12}^{-1}+y_{3}^{2} M_{3}^{-1}\end{array}\right]$ \\
\hline $\mathrm{C}$ & $\left.\begin{array}{lll}1 & \lambda & \lambda \\
\lambda & 1 & 1 \\
\lambda & 1 & 1\end{array}\right]$ & $\left.\begin{array}{lll}y_{1} & & \\
& 1 & \\
& & \\
& & 1\end{array}\right]$ & $\begin{array}{lll}1 & \rho & \rho \\
\rho & 1 & \rho \\
\rho & \rho & 1\end{array}$ & $\begin{array}{ccc}y_{1}^{2} M_{1}^{-1} & \checkmark & \checkmark \\
y_{i}^{2} M_{i}^{-1} \lambda+y_{1}\left(y_{2}+y_{3}\right) M_{13}^{-1} \rho & y_{2}^{2} M_{2}^{-1}+y_{3}^{2} M_{3}^{-1} & \sqrt{ } \\
y_{i}^{2} M_{i}^{-1} \lambda+y_{1}\left(y_{2}+y_{3}\right) M_{13}^{-1} \rho & y_{2}^{2} M_{2}^{-1}+y_{3}^{2} M_{3}^{-1} & y_{2}^{2} M_{2}^{-1}+y_{3}^{2} M_{3}^{-1}\end{array}$ \\
\hline $\mathbf{D}$ & $\left.\begin{array}{lll}1 & \lambda & \lambda \\
\lambda & 1 & 1 \\
\lambda & 1 & 1\end{array}\right]$ & $\begin{array}{ll}y_{2} & \\
& 1\end{array}$ & {$\left[\begin{array}{lll}1 & \rho & \rho \\
\rho & 1 & 1 \\
\rho & 1 & 1\end{array}\right]$} & $\begin{array}{ccc}y_{1}^{2} M_{1}^{-1} & \checkmark & \checkmark \\
y_{i}^{2} M_{i}^{-1} \lambda+y_{1}\left(y_{2}+y_{3}\right) M_{13}^{-1} \rho & y_{2}^{2} M_{2}^{-1}+y_{3}^{2} M_{3}^{-1} & \sqrt{ } \\
y_{i}^{2} M_{i}^{-1} \lambda+y_{1}\left(y_{2}+y_{3}\right) M_{13}^{-1} \rho & y_{2}^{2} M_{2}^{-1}+y_{3}^{2} M_{3}^{-1} & y_{2}^{2} M_{2}^{-1}+y_{3}^{2} M_{3}^{-1}\end{array}$ \\
\hline $\mathbf{E}$ & $\left.\begin{array}{lll}1 & \lambda & \lambda \\
\lambda & 1 & \lambda \\
\lambda & \lambda & 1\end{array}\right]$ & 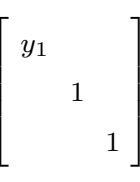 & {$\left[\begin{array}{lll}1 & \rho & \rho \\
\rho & 1 & 1 \\
\rho & 1 & 1\end{array}\right]$} & $\left.\begin{array}{ccc}y_{1}^{2} M_{1}^{-1} & \sqrt{ } & \sqrt{ } \\
\left(y_{1}^{2} M_{1}^{-1}+y_{2} y_{23} M_{23}^{-1}\right) \lambda+y_{1} y_{2} M_{123}^{-1} \rho & y_{2}^{2} M_{23}^{-1} & \sqrt{ } \\
\left(y_{1}^{2} M_{1}^{-1}+y_{3} y_{23} M_{23}^{-1}\right) \lambda+y_{1} y_{3} M_{123}^{-1} \rho & y_{2} y_{3} M_{23}^{-1} & y_{3}^{2} M_{23}^{-1}\end{array}\right]$ \\
\hline $\mathbf{F}$ & $\left.\begin{array}{lll}1 & 1 & \lambda \\
1 & 1 & 1 \\
1 & 1 & 1\end{array}\right]$ & $\begin{array}{lll}y_{1} & & \\
& & \\
& y_{2} & \\
& & 1\end{array}$ & {$\left[\begin{array}{lll}1 & 1 & \rho \\
1 & 1 & \rho \\
\rho & \rho & 1\end{array}\right]$} & $\left.\begin{array}{ccc}y_{12}^{2} M_{12}^{-1} & \sqrt{ } & \checkmark \\
y_{12}^{2} M_{12}^{-1} & y_{12}^{2} M_{12}^{-1}+y_{3}^{2} M_{3}^{-1} & \checkmark \\
y_{12}^{2} M_{12}^{-1} & y_{12}^{2} M_{12}^{-1}+y_{3}^{2} M_{3}^{-1} & y_{12}^{2} M_{12}^{-1}+y_{3}^{2} M_{3}^{-1}\end{array}\right]$ \\
\hline G & $\left.\begin{array}{lll}1 & 1 & \lambda \\
1 & 1 & 1 \\
1 & 1 & 1\end{array}\right]$ & $\begin{array}{lll}y_{1} & & \\
& & \\
& y_{2} & \\
& & 1\end{array}$ & {$\left[\begin{array}{lll}1 & \rho & \rho \\
\rho & 1 & \rho \\
\rho & \rho & 1\end{array}\right]$} & $\left.\begin{array}{ccc}y_{1}^{2} M_{1}^{-1}+y_{2}^{2} M_{2}^{-1} & \sqrt{ } & \sqrt{ } \\
y_{1}^{2} M_{1}^{-1}+y_{2}^{2} M_{2}^{-1} & y_{i}^{2} M_{i}^{-1} & \sqrt{ } \\
y_{1}^{2} M_{1}^{-1}+y_{2}^{2} M_{2}^{-1} & y_{i}^{2} M_{i}^{-1} & y_{i}^{2} M_{i}^{-1}\end{array}\right]$ \\
\hline
\end{tabular}


condition with $y_{2} \sim y_{3}$ ruled out a large angle at $R_{12}$, as shown in Table 【. When $y_{2} \ll y_{3}$, $\left(\mathbb{R}_{2}, \mathbb{L}_{1}\right)$ for Model B, $\left(\mathbb{R}_{4}, \mathbb{L}_{1}\right)$ for Model D, $\left(\mathbb{R}_{2}, \mathbb{L}_{3}\right)$ for Model $\mathbf{F},\left(\mathbb{R}_{4}, \mathbb{L}_{3}\right)$ for Model $\mathbf{G}$ can predict the large mixing angle for atmospheric neutrinos and $\mathrm{CHOOZ}$ bound of reactor neutrinos. See Appendix.

Thus, the structures of Yukawa matrices that satisfy three constraints:

(i) the out-of-equilibrium condition of lepton number violating processes,

(ii) the upper bound of the branching ratio of the rare decay $\mu \rightarrow e \gamma$,

(iii) the large mixing angle in atmospheric neutrino oscillation,

must be one of the 7 combinations, Models A to $\mathbf{G}$ in Tables II and III, which establish the relative dominance of $y_{2}$ and $y_{3}$, and the approximate magnitudes of transformation matrices, $|\mathbb{R}|$ and $|\mathbb{L}|$. In Table $\mathbb{I V}$ is the list of models of Yukawa matrices and the light neutrino mass matrix divided by $v^{2}$. Models are specified in terms of two unitary transformations and the relative dominance in eigenvalues as summary of Tables II and III. The notations are introduced in Eqs.(39), (41), (42), and (A1)-(A3). The entry marked with $\sqrt{ }$ represents the corresponding symmetric elements. There are two types of spectrum of eigenvalues, $y_{1} \ll y_{2}, y_{1} \sim y_{2} \ll y_{3} \sim 1$ and $y_{1} \ll y_{2} \sim y_{3} \sim 1$, which correspond to Case -a,-c and Case - $\mathbf{b}$ introduced for Table 【 respectively.

In the Appendix, Table $\nabla$ describes in detail which constraints keep certain entries in Tables 【 and II from being eligible models. The Table $\nabla$ in Appendix A.2 shows obviously that the cases prohibited by the constraint (ii) are already prohibited by the constraint (iii). The constraint (ii) turns out as a redundant one in checking the eligibility of Yukawa matrix to known leptonic phenomenology. In other words, the structure of Yukawa matrix which can derive the light neutrino mass matrix through see-saw mechanism always appear to give rise to $\operatorname{Br}(\mu \rightarrow e \gamma)$ below the present experimental limit in SUSY theories with large $\tan \beta$ and universal slepton mass at GUT scale. It is possible to pull out a constraint to narrow down the estimation of heavy neutrino masses from LFV through the see-saw mechanism. 


\section{MODELS AND A LINK BETWEEN LEPTOGENESIS AND LOW-ENERGY NEUTRINOS}

\section{A. Application of bi-unitary parametrization}

The light neutrino mass matrix defined through the seesaw mechanism in Eq.(37) is diagonalized as

$$
\mathbb{V} m_{L} \mathbb{V}^{T}=-v_{2}^{2} \mathbb{L} \mathcal{Y}_{D} \mathbb{R}^{\dagger} M_{R}^{-1} \mathbb{R}^{*} \mathcal{Y}_{D}^{T} \mathbb{L}^{T}
$$

where $\mathbb{V}$ is MNS light neutrino transformation matrix and $m_{L}$ is a diagonal matrix with mass eigenvalues $m_{i}$. The equation of $3 \times 3$ symmetric complex matrices in Eq.(43) consists of 12 equations of parameters, while its right hand side consists of 18 unknowns: 3 angles and 3 phases in $\mathbb{L}, 3$ angles and 3 phases in $\mathbb{R}, 3$ eigenvalues in $\mathcal{Y}_{D}$, and 3 heavy neutrino masses in $M_{R}$. Once one makes a choice out of the 7 models model $\mathbf{A}$ to model $\mathbf{G}$ specified

in Tables II and III one can draw the boundary of eligible parameter space starting the 6 angles, large or small. Even though it is supposed that the number of the free parameters reduces to 12 for 12 equations, considering 6 angles in $|\mathbb{R}|$ and $|\mathbb{L}|$ fixed, solving Eq.(43) yet imbeds a great deal of ambiguity, since CP phases in MNS matrix and individual mass eigenvalues in left-hand side can barely be said as fixed.

There are a few approaches to make improvement in solving the see-saw mechanism depending on models. First, one can rely on theories with additional symmetries to reduce the number of parameters. For example, in case of Model G, a flavor symmetry like $U(1)$ can be utilized to simplify the mechanism in Eq. (43), because the charges of the flavor symmetry assigned to $\nu_{R i}\left(\ell_{i}\right)$ can result in the common constraint on the side of right (left) mixing in Yukawa matrix and the structure of right (left) neutrino mass matrix. The structure of Yukawa matrix in Model E can fit in a theory of grand-unified-type symmetry between leptons and quarks, if $\mathbb{L}_{1}$ is close to CKM matrix. Among eigenvalues of Yukawa matrix and 3 heavy masses, there are a few parameters which can be fixed by hands motivated from a higher-rank symmetry. They can be $y_{3}$ of order one and/or $M_{3}$ of GUT scale. The $y_{3} \sim 1$ has been used in a number of times since Eq.(27), as well as $y_{2} \sim y_{3}$ in particular models.

Another possible approach can be collecting more phenomenological constraints involved with neutrino Yukawa couplings to diminish the number of the eligible structure of Yukawa 
matrix, aiming the survival of only one natural structure. The classification derived based on bi-unitary transformations is useful in both approaches. That is, the models parameterized using bi-unitary transformation of Yukawa matrix can be easily tested for the compatibility to additional symmetries as proposed in the previous paragraph. The bi-unitary parametrization is useful also to examine the eligibility to additional phenomenology. In describing a process, the loop contributions of Yukawa couplings consist of a hermitian operator $\mathcal{Y}^{\dagger} \mathcal{Y}$ or $\mathcal{Y Y}^{\dagger}$. It is always possible to rephrase operator in terms of the minimal number of parameters, the pair of $\left(\mathcal{Y}_{D}, \mathbb{R}\right)$ or the pair of $\left(\mathcal{Y}_{D}, \mathbb{L}\right)$ without any mixing of the two transformations. Thus, application of an additional constraint will be simply the re-examination of the 7 models characterized by eigenvalues of Yukawa matrix, left and right transformations.

\section{B. A model of neutrino masses}

The description of leptogenesis requires fine understanding of CP phases in Yukawa matrix as well as CP phases in light neutrino mass matrix in order to secure a sufficient amount of CP asymmetry from the decays of heavy Majorana neutrinos. In lepton sector, however, it is not practical to regard the CP phases at low-energy as known parameters. Rather, in analogy of quark sector, the size of a real mixing angle can be realized as the source of certain amount of $\mathrm{CP}$ violation before the derivation of detailed imaginary parts. Many models proposed so far took simply order one contribution from CP phase.

As far as the detailed discussion on $\mathrm{CP}$ phases is set aside, Models $\mathbf{A}, \mathbf{D}$, and $\mathbf{E}$ with a large angle at $R_{23}$ can provide optimal scenario for the sufficient amount of CP asymmetry, accordingly lepton asymmetry, Eq.(15). There are a number of cases listed in Table \which can enhance the CP asymmetry $\epsilon_{1}$, if $\left|R_{23}\right|$ becomes close to one, so as to reach its maximum value.

$$
\epsilon_{1} \sim 10^{-1} y_{3}^{2}\left|R_{23}\right|^{2} \frac{M_{1}}{M_{2}} \sim 10^{-1} \frac{M_{1}}{M_{2}}
$$

It is worthy of attention that large mixing angles in low-energy originate entirely from the large mixings in heavy neutrinos in Model E. 
The Yukawa matrix in Model $\mathbf{E}$ is

$$
\left|\mathcal{Y}_{N}\right| \equiv\left(\begin{array}{ccc}
1 & \lambda & \lambda \\
\lambda & 1 & \lambda \\
\lambda & \lambda & 1
\end{array}\right)\left(\begin{array}{lll}
y_{1} & 0 & 0 \\
0 & 1 & 0 \\
0 & 0 & 1
\end{array}\right)\left(\begin{array}{lll}
1 & \rho & \rho \\
\rho & 1 & 1 \\
\rho & 1 & 1
\end{array}\right),
$$

where $y_{2} \sim y_{3} \sim 1, y_{1} \ll y_{2}$. It is possible that the Model $\mathbf{E}$ reduces to the model with two-generation of heavy neutrinos as the large mixing angles become maximal and the degeneracy in eigenvalues becomes exact. In such limit, the leading contribution to $\epsilon_{1}$ does not any longer come from $R_{23}$. The matrix in Eq.(45) was introduced in the basis the mass matrix of righthanded neutrinos is diagonal. Now, a new basis can be chosen in such a way that Yukawa matrix is symmetric. Let the Yukawa matrix in Model $\mathbf{E}$ in the new basis be

$$
\left|\mathcal{Y}_{N}^{\prime}\right| \equiv\left(\begin{array}{ccc}
1 & \lambda & \lambda \\
\lambda & 1 & \lambda \\
\lambda & \lambda & 1
\end{array}\right)\left(\begin{array}{ccc}
y_{1} & 0 & 0 \\
0 & 1 & 0 \\
0 & 0 & 1
\end{array}\right)\left(\begin{array}{ccc}
1 & \rho^{\prime} & \rho^{\prime} \\
\rho^{\prime} & 1 & \rho^{\prime} \\
\rho^{\prime} & \rho^{\prime} & 1
\end{array}\right) .
$$

Then, the righthanded neutrino mass matrix in Eq.(43) will have a structure with a large mixing angle in the new basis,

$$
\begin{aligned}
& M_{R}^{\prime} \sim \mathbb{R}^{\prime} M_{R} \mathbb{R}^{T}, \\
& \mathbb{R}^{\prime} \equiv \mathbb{R}_{\text {new }}^{*} \mathbb{R}_{\text {old }}^{T},
\end{aligned}
$$

where $\mathbb{R}_{\text {old }}$ is the right transformation to diagonalize the Yukawa matrix from the old basis in Eq.(45), while $\mathbb{R}_{\text {new }}$ is the one for the diagonalization from the new basis in Eq.(46). The Model E can represent a model in left-right symmetry, in other words, the model shows a well-balanced correspondence between low energy and high energy over see-saw mechanism, since the Yukawa matrix is symmetric and two Majorana neutrino mass matrices are very much of analogy.

The difference of the lepton sector from the quark sector was recognized from the large mixing angles. In this scenario, the dissimilarity in the two sectors can be understood as originated from the existence of massive singlet neutrinos, whereas nothing like that is in the quark sector. There might have been dynamics of heavy neutrinos in the early universe to generate the large mixing angles of which become later the source of the large mixing angles of light neutrinos. It is shown in Eq.(44) that the leptogenesis is optimized. The Eqs. (46)-(47) help the see-saw mechanism look more meaningful with this model in a theory of left-right symmetry. 


\section{Leptogenesis and neutrino large mixing angles}

Before concluding the section, we give a some thought on the role of the see-saw mechanism, expressing $\mathrm{CP}$ asymmetry in terms of light neutrino parameters in a model-independent way. Using solutions to Eq.(43) for a chosen model, one can express the CP asymmetry in a proper way to replace the heavy neutrino masses in terms of low energy neutrino observables and parameters in Yukawa matrix. The diagonal neutrino mass matrix in Eq.(43) can be rephrased as

$$
m_{L}=-v_{2}^{2} \mathbb{V}^{T} \tilde{\mathcal{Y}}_{N} M_{R}^{-1} \mathbb{P}^{2} \tilde{\mathcal{Y}}_{N}^{T} \mathbb{V}
$$

where $\tilde{\mathcal{Y}}_{N} \equiv \mathcal{Y}_{N} \mathbb{P}^{\dagger}$ is defined in such a way that phases in $\mathbb{P}$ do not appear explicitly in $\tilde{\mathcal{Y}}_{N}$ as hidden in Eq.(201). Alternatively,

$$
M_{k} \mathbb{P}_{k}^{-2}=-v_{2}^{2} \sum_{k^{\prime}} \frac{1}{m_{k^{\prime}}}\left(\mathbb{V}^{T} \tilde{\mathcal{Y}}_{N}\right)_{k^{\prime} k}{ }^{2}
$$

the imaginary parts of whose inverse are obtained as

$$
\begin{aligned}
& \frac{\sin 2 \phi_{1}}{M_{2}}=\frac{1}{v_{2}^{2}}\left(\sum_{k} \frac{1}{m_{k}} \operatorname{Im}\left[\mathbb{V}^{T} \tilde{\mathcal{Y}}_{N}\right]_{k 2}{ }^{2}\right)^{-1}, \\
& \frac{\sin 2 \phi_{2}}{M_{3}}=\frac{1}{v_{2}^{2}}\left(\sum_{k} \frac{1}{m_{k}} \operatorname{Im}\left[\mathbb{V}^{T} \tilde{\mathcal{Y}}_{N}\right]_{k 3}{ }^{2}\right)^{-1},
\end{aligned}
$$

while

$$
M_{1}=\frac{1}{v_{2}^{2}} \sum_{k} \frac{1}{m_{k}}\left[\mathbb{V}^{T} \tilde{\mathcal{Y}}_{N}\right]_{k 1}^{2}
$$

When the value of the rectangular brackets of the above equation is of order one, the term with $m_{1}^{-1}$ is guaranteed to be a significantly leading contribution compared to the other terms with $m_{2}^{-1}$ or $m_{3}^{-1}$ inside the summation, resulting in the enhancement in the scale of $M_{1}$. In Ref.[17], it was examined that large mixing angles in MNS matrix can predict the small dilution mass preferred by the out-of-equilibrium condition in a model. In the right hand side of Eq.(21) $\sin 2 \phi_{1}\left(M_{1} / M_{2}\right)$ and $\sin 2 \phi_{2}\left(M_{1} / M_{3}\right)$ can be re-parameterized only in terms of Yukawa matrix and light neutrino masses and mixings using Eqs.(151) and (52). The enhancement of $M_{1}$ contributed from large mixing angles in Eq.(52) results in the enhancement in the amount of the CP violation in Eq.(21). 


\section{SUMMARY}

The CP asymmetry and the out-of-equilibrium condition of lepton flavor violating process are analyzed in terms of eigenvalues and right mixing angles. In the out-of-equilibrium condition, there are significant implications. The mixing element $\left|\mathbb{R}_{13}\right|$ should be small with an upper bound, Eq.(28), and two eigenvalues $y_{1}$ and $y_{3}$ cannot be of similar size, Eq.(29). Depending on which term in expression of the condition actually leads the condition or depending on whether eigenvalues are of hierarchy or of similar size, the magnitude of right transformation is characterized differently, as shown in Table I. Examining all the possible models, we found the model independent maximal CP asymmetry to be $10^{-1} M_{1} / M_{2}$, Eq.(32). Besides thermal out-of-equilibrium condition, we examined also the upper bound of a LFV decay and mixing angles in neutrino oscillations to constrain all kinds of parameters, i.e., left and right mixing elements and eigenvalues. We found that only the seven models can characterize phenomenologically eligible Yukawa matrices.

The constraint from the LFV did not exclude any choice of parameter region addition to the category excluded by two other constraints. In other words, the survival of only seven models is the outcome of the application of two constraints: the out-of-equilibrium condition and data of neutrino oscillation. In Table $\mathbb{V}$, the determination of eligible models from the application of certain constraints is specified. The bi-unitary parametrization is useful for model test of the compatibility to additional symmetries and the eligibility to additional phenomenology. The Model $\mathbf{E}$ is appealing in a sense that the size of the CP asymmetry is maximized and the number of free parameters can be reduced based on left-right symmetry.

\section{Acknowledgments}

The author wishes to express her thanks to Mike Berger for his advice. She thanks Eung-Jin Chun for the discussion and suggestion on this work during her stay at KIAS. She thanks Chuan-Hung Chen since a number of parts were progressed from discussion with him. She thanks Yasutaka Takanishi for his helpful comment on LFV. 


\section{APPENDIX A: REMARKS ON TABLES II AND III}

\section{Recovery of small angle contribution in transformations}

In Tables [I] and [II, the elements of matrices determined from small angles in transformations are not specified. (They are indicated by a *.) The Models $\mathbf{A}, \mathbf{C}$, and $\mathbf{E}$ include elements, the leading order of which is first order in $\lambda$ or $\rho$, while other models have their leading order all zeroth in $\lambda$ and $\rho$. The small values are not all of the same order of magnitude in a transformation matrix. However, different small angles do not distinguish because the comparison of their sizes is not necessary to examine the eligibility of a model to given constraints in this paper. With $\rho$ 's and $\lambda$ 's in $\mathbb{R}$ 's and $\mathbb{L}$ 's, respectively, the possible symmetric structures of matrix $m_{\nu} / v_{2}^{2}$ of Models A, C, and $\mathbf{E}$ will be as follow;

\section{Model A}

$$
\left[\begin{array}{ccc}
y_{1}^{2} M_{1}^{-1} & \sqrt{ } & \sqrt{ } \\
\left(y_{1}^{2} M_{1}^{-1}+y_{23}^{2} M_{23}^{-1}\right) \lambda+y_{1} y_{23} M_{123}^{-1} \rho & y_{23}^{2} M_{23}^{-1} & \sqrt{ } \\
\left(y_{1}^{2} M_{1}^{-1}+y_{23}^{2} M_{23}^{-1}\right) \lambda+y_{1} y_{23} M_{123}^{-1} \rho & y_{23}^{2} M_{23}^{-1} & y_{23}^{2} M_{23}^{-1}
\end{array}\right]
$$

Model C

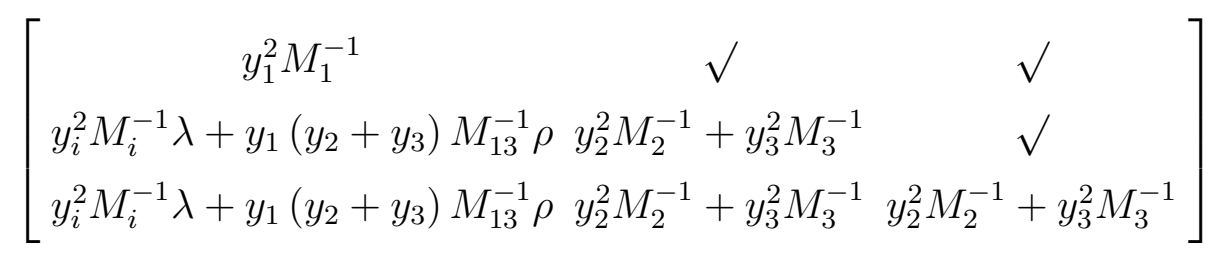

\section{Model E}

$$
\left[\begin{array}{ccc}
y_{1}^{2} M_{1}^{-1} & \sqrt{ } & \sqrt{ } \\
\left(y_{1}^{2} M_{1}^{-1}+y_{2} y_{23} M_{23}^{-1}\right) \lambda+y_{1} y_{2} M_{123}^{-1} \rho & y_{2}^{2} M_{23}^{-1} & \sqrt{ } \\
\left(y_{1}^{2} M_{1}^{-1}+y_{3} y_{23} M_{23}^{-1}\right) \lambda+y_{1} y_{3} M_{123}^{-1} \rho & y_{2} y_{3} M_{23}^{-1} & y_{3}^{2} M_{23}^{-1}
\end{array}\right]
$$

where $i=1-3$ and the entry marked by $\sqrt{ }$ represents the corresponding symmetric elements.

The 1-2 sector in light neutrino mass needs to be examined to see whether a model is consistent with for the large mixing angle of solar neutrino oscillation. In order for a mass matrix to have the large mixing angle between the first and the second generations, the 1-2 element of the matrix $m_{\nu 12}$ should be not smaller than the 1-1 element $m_{\nu 11}$. If one takes a 
TABLE V: The specification of constraints which in fact rule out certain types of Yukawa matrices represented by a $\mathbb{R}$, a $\mathbb{L}$, and the comparison of $y_{2}$ and $y_{3}$. The $\mathrm{C} 1, \mathrm{C} 2, \mathrm{C} 3$, and $\mathrm{C} 4$ are the indices of the constraints explained in Section A.2. For example, Yukawa matrix characterized by $\mathbb{R}_{1}, \mathbb{L}_{1}$ and $y_{2} \ll y_{3}$ are forbidden due to the constraint $\mathrm{C} 4$.

\begin{tabular}{lccccc}
\hline \hline & & $\mathbb{L}_{1}$ & $\mathbb{L}_{2}$ & $\mathbb{L}_{3}$ & $\mathbb{L}_{4}$ \\
\hline $\mathbb{R}_{1}$ & $y_{2} \ll y_{3}$ & C4 & C3 & C4 & C3 \\
& $y_{2} \sim y_{3}$ & Model A & C2 C3 & C2 C3 & Model E \\
\hline $\mathbb{R}_{2}$ & $y_{2} \ll y_{3}$ & Model B & C3 & Model F & C3 \\
& $y_{2} \sim y_{3}$ & C1 & C1 C2 C3 & C1 C2 & C1 C3 \\
\hline $\mathbb{R}_{3}$ & $y_{2} \ll y_{3}$ & C4 & C3 & C4 & C3 \\
& $y_{2} \sim y_{3}$ & C1 & C1 C2 C3 & C1 C2 & C1 \\
\hline $\mathbb{R}_{4}$ & $y_{2} \ll y_{3}$ & Model C & C3 & Model G & C3 \\
& $y_{2} \sim y_{3}$ & Model D & C2 C3 & C2 C3 & C3 \\
\hline \hline
\end{tabular}

hierarchical neutrino mass spectrum $m_{2} \sim \sqrt{\triangle m_{\odot}^{2}}$ and $m_{3} \sim \sqrt{\triangle m_{\text {atm }}^{2}}$, then the structure of neutrino mass matrix should require

$$
\frac{m_{\nu 12}}{m_{\nu 22}} \sim \sqrt{\frac{\triangle m_{\odot}^{2}}{\triangle m_{a t m}^{2}}},
$$

i.e., the ratio between the two elements should be suppressed by one order of magnitude 33]. The $m_{\nu} / v_{2}^{2}$ matrices for the three models in Eqs. (A1)-(A3) show that there can be parameter region which allows large mixing angle solution for solar neutrino oscillation as well as the large mixing angles of atmospheric neutrinos and satisfies the CHOOZ bound of the reactor neutrinos.

\section{Application of constraints}

The exclusion of certain types of Yukawa structures is explained in Table $\nabla$ by specifying the constraint violated by each particular structure. The constraint $\mathrm{C} 1$ is equivalent to the constraint (i) in listed in Section IV. The out-of-equilibrium condition of the lepton number violating process, Eq.(24), does not allow a structure of Yukawa matrix with both $y_{2} \sim y_{3}$ and $R_{12} \sim 1$. 
The constraint C2 is equivalent to the constraint (ii) in Section IV. The suppression in $\left(\mathcal{Y}_{N} \mathcal{Y}_{N}^{\dagger}\right)_{21}$ for $B R(\mu \rightarrow e \gamma)<1.2 \times 10^{-11}$ does not allow the structure of Yukawa matrix with both $y_{2} \sim y_{3}$ and $L_{12} \sim 1$.

The constraint C3 is equivalent to the constraint (iii) in Section IV. The phenomenological constraints for light neutrino experiments require the structure of mass matrix to have $m_{\nu 13} \ll m_{\nu 23} \sim m_{\nu 33}$.

The last constraint $\mathrm{C} 4$ is the requirement for Yukawa matrix not to involve fine tuning. It is not appropriate to assume any possible fine tuning in Yukawa structure since the comparison of parameters was done whether it is hierarchical or nearly degenerate, whereas it is unavoidable to assume some possible fine tuning in consideration with light neutrino masses due to the coexistence of large mixing angles and hierarchical mass spectrum in its symmetric structure. In the Yukawa matrices, if $y_{2} \ll y_{3}$ when $L_{23} \sim 1$ and $R_{23} \sim 1$, such a small eigenvalue $y_{2}$ can be obtained only by fine tuning. For that reason, the cases with $L_{23} \sim R_{23} \sim 1, y_{2} \ll y_{3}$ are ruled out. In actual model construction with respect to data, however, the structures restricted by $\mathrm{C} 4$ can be regarded rather positively, if the fine-tuning is the only way to accommodate data. If one allows tuning with $L_{23} \sim R_{23} \sim 1, y_{2} \ll y_{3}$, there can be four more eligible models which are marked by C4 in Table $\mathrm{V}$.

[1] A. D. Sakharov, Pisma Zh. Eksp. Teor. Fiz. 5, 32 (1967) [JETP Lett. 5, 24 (1967 SOPUA,34,392-393.1991 UFNAA,161,61-64.1991)].

[2] V. A. Kuzmin, V. A. Rubakov and M. E. Shaposhnikov, Phys. Lett. B 155, 36 (1985).

[3] M. Fukugita and T. Yanagida, Phys. Lett. B 174, 45 (1986).

[4] S. Y. Khlebnikov and M. E. Shaposhnikov, Nucl. Phys. B 308, 885 (1988).

[5] J. A. Harvey and M. S. Turner, Phys. Rev. D 42, 3344 (1990).

[6] K. Hagiwara et al. [Particle Data Group Collaboration], Phys. Rev. D 66, 010001 (2002).

[7] E. W. Kolb and M. S. Turner, "The Early Universe," Addison-Wesley, 1990

[8] E. W. Kolb and S. Wolfram, Nucl. Phys. B 172, 224 (1980) [Erratum-ibid. B 195, 542 (1982)].

[9] M. A. Luty, Phys. Rev. D 45, 455 (1992).

[10] M. Flanz, E. A. Paschos and U. Sarkar, Phys. Lett. B 345, 248 (1995) [Erratum-ibid. B 382, 447 (1996)] arXiv:hep-ph/9411366. 
[11] L. Covi, E. Roulet and F. Vissani, Phys. Lett. B 384, 169 (1996) arXiv:hep-ph/9605319.

[12] W. Buchmuller and M. Plumacher, Phys. Lett. B 431, 354 (1998) arXiv:hep-ph/9710460.

[13] A. Pilaftsis, Phys. Rev. D 56, 5431 (1997) arXiv:hep-ph/9707235. A. Pilaftsis, Int. J. Mod. Phys. A 14, 1811 (1999) arXiv:hep-ph/9812256.

[14] A. S. Joshipura, E. A. Paschos and W. Rodejohann, JHEP 0108, 029 (2001) arXiv:hep-ph/0105175 . A. S. Joshipura, E. A. Paschos and W. Rodejohann, Nucl. Phys. B 611, 227 (2001) arXiv:hep-ph/0104228.

[15] W. Buchmuller and M. Plumacher, Int. J. Mod. Phys. A 15, 5047 (2000) arXiv:hep-ph/0007176.

[16] W. Buchmuller and T. Yanagida, Phys. Lett. B 445, 399 (1999) arXiv:hep-ph/9810308. M. S. Berger and B. Brahmachari, Phys. Rev. D 60, 073009 (1999) arXiv:hep-ph/9903406. M. S. Berger, Phys. Rev. D 62, 013007 (2000) arXiv:hep-ph/9906490. M. Hirsch and S. F. King, Phys. Rev. D 64, 113005 (2001) arXiv:hep-ph/0107014. T. Hambye, E. Ma and U. Sarkar, Nucl. Phys. B 602, 23 (2001) arXiv:hep-ph/0011192. D. Falcone and F. Tramontano, Phys. Rev. D 63, 073007 (2001) arXiv:hep-ph/0011053. E. J. Chun and S. K. Kang, Phys. Rev. D 63, 097902 (2001) arXiv:hep-ph/0001296. H. B. Nielsen and Y. Takanishi, Phys. Lett. B 507, 241 (2001) arXiv:hep-ph/0101307. D. Falcone, Phys. Rev. D 65, 077301 (2002) arXiv:hep-ph/0111176. Z. z. Xing, Phys. Lett. B 545, 352 (2002) arXiv:hep-ph/0206245.

[17] M. S. Berger and K. Siyeon, Phys. Rev. D 65, 053019 (2002). arXiv:hep-ph/0110001.

[18] M. Gell-Mann, P. Ramond and R. Slansky, Print-80-0576 (CERN). T. Yanagida, Proceedings Of The Workshop On The Unified Theory And The Baryon Number In The Universe, R. N. Mohapatra and G. Senjanovic, Phys. Rev. Lett. 44, 912 (1980).

[19] G. C. Branco, T. Morozumi, B. M. Nobre and M. N. Rebelo, Nucl. Phys. B 617, 475 (2001) arXiv:hep-ph/0107164.

[20] M. N. Rebelo, Phys. Rev. D 67, 013008 (2003) arXiv:hep-ph/0207236.

[21] W. Buchmuller, P. Di Bari and M. Plumacher, Nucl. Phys. B 643, 367 (2002) arXiv:hep-ph/0205349.

[22] S. Davidson and A. Ibarra, Phys. Lett. B 535, 25 (2002) arXiv:hep-ph/0202239.

[23] P. H. Frampton, S. L. Glashow and T. Yanagida, Phys. Lett. B 548, 119 (2002) arXiv:hep-ph/0208157. T. Endoh, S. Kaneko, S. K. Kang, T. Morozumi and M. Tanimoto, Phys. Rev. Lett. 89, 231601 (2002) arXiv:hep-ph/0209020. G. C. Branco, R. Gonzalez Felipe, 
F. R. Joaquim, I. Masina, M. N. Rebelo and C. A. Savoy, arXiv:hep-ph/0211001, M. Raidal and A. Strumia, Phys. Lett. B 553, 72 (2003) arXiv:hep-ph/0210021. J. I. Silva-Marcos, arXiv:hep-ph/0212089.

[24] S. Kaneko and M. Tanimoto, Phys. Lett. B 551, 127 (2003) arXiv:hep-ph/0210155.

[25] S. Fukuda et al. [Super-Kamiokande Collaboration], Phys. Rev. Lett. 86, 5656 (2001) arXiv:hep-ex/0103033.

[26] M. Apollonio et al. [CHOOZ Collaboration], Phys. Lett. B 466, 415 (1999) arXiv:hep-ex/9907037.

[27] Z. Maki, M. Nakagawa and S. Sakata, Prog. Theor. Phys. 28, 870 (1962).

[28] S. Pascoli, S. T. Petcov and W. Rodejohann, arXiv:hep-ph/0302054.

[29] S. T. Petcov, Sov. J. Nucl. Phys. 25, 340 (1977) [Yad. Fiz. 25, 641 (1977 ERRAT,25,698.1977 ERRAT,25,1336.1977)]. T. P. Cheng and L. F. Li, Phys. Rev. D 16, 1425 (1977). W. J. Marciano and A. I. Sanda, Phys. Lett. B 67, 303 (1977). B. W. Lee and R. E. Shrock, Phys. Rev. D 16, $1444(1977)$.

[30] J. Hisano, T. Moroi, K. Tobe and M. Yamaguchi, Phys. Rev. D 53, 2442 (1996) arXiv:hep-ph/9510309.

[31] J. A. Casas and A. Ibarra, Nucl. Phys. B 618, 171 (2001) arXiv:hep-ph/0103065. T. Hambye, arXiv:hep-ph/0210048.

[32] J. R. Ellis, J. Hisano, M. Raidal and Y. Shimizu, Phys. Rev. D 66, 115013 (2002) arXiv:hep-ph/0206110. J. R. Ellis and M. Raidal, Nucl. Phys. B 643, 229 (2002) arXiv:hep-ph/0206174. D. Falcone, Mod. Phys. Lett. A 17, 2467 (2002) arXiv:hep-ph/0207308. G. Cvetic, C. Dib, C. S. Kim and J. D. Kim, Phys. Rev. D 66, 034008 (2002) arXiv:hep-ph/0202212.

[33] M. C. Gonzalez-Garcia, arXiv:hep-ph/0211054 